\title{
Immuno-Oncology Therapy for Hepatocellular Carcinoma: Current Status and Ongoing Trials
}

Masatoshi Kudo

Department of Gastroenterology and Hepatology, Kindai University Faculty of Medicine, Osaka-Sayama, Japan

Prof. M. Kudo

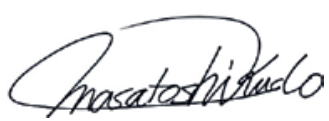

Editor Liver Cancer

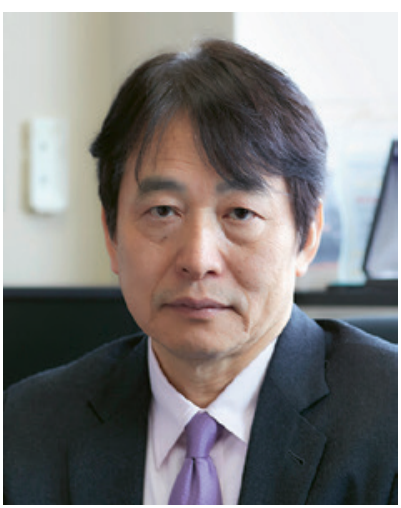

\section{Introduction}

Several anti-PD-1, anti-PD-L1, and anti-CTLA-4 antibodies (Table 1) are currently being investigated, alone and in combination, for the treatment of hepatocellular carcinoma (HCC), and this field is advancing rapidly. This Editorial will review the latest data on the use of immune checkpoint inhibitors in HCC, including combination strategies.

\section{Cancer Immunity Cycle}

The major histocompatibility complex on antigen-presenting cells recognizes tumorassociated antigens on the surface of cancer cells and presents the antigen to T-cell receptors on immature T cells upon migration of antigen-presenting cells to the lymph nodes. Immature $\mathrm{T}$ cells are not activated by antigen stimulation (first signal) alone, but also require an activating costimulatory signal (second signal). The second signal is provided by the binding of 
Kudo: Immuno-Oncology Therapy for Hepatocellular Carcinoma: Current Status and Ongoing Trials

Table 1. Immune checkpoint inhibitors in HCC [32]

\begin{tabular}{|c|c|c|c|c|c|c|}
\hline Target cell & Molecule & Develop. code & Drug name & TM name & Antibody class & Company \\
\hline \multirow[t]{4}{*}{$\mathrm{T}$ cell } & PD-1 & $\begin{array}{l}\text { BMS-36558 } \\
\text { ONO-4538 }\end{array}$ & Nivolumab & Opdivo & Fully human IgG4 antibody & ONO/BMS \\
\hline & PD-1 & MK-4375 & Pembrolizumab & Keytruda & Humanized IgG4 antibody & Merck \\
\hline & PD-1 & BGB-A317 & Tislelizumab & Not approved & Humanized IgG4 antibody & BeiGene \\
\hline & PD-1 & SHR-1210 & Camrelizumab & Not approved & Humanized IgG4 antibody & $\begin{array}{l}\text { Jiangsu HengRui } \\
\text { Medicine }\end{array}$ \\
\hline \multirow[t]{3}{*}{ Tumor cell } & PD-L1 & MPDL3280A & Atezolizumab & Tecentriq & Fully human IgG1 antibody & Roche \\
\hline & PD-L1 & MEDI4736 & Durvalumab & Imfinzi & humanized IgG1 antibody & AstraZeneca \\
\hline & PD-L1 & MSB-0010718C & Avelumab & Barencio & Humanized IgG1 antibody & Merck-Serono \\
\hline \multirow[t]{2}{*}{$\mathrm{T}$ cell } & CTLA-4 & BMS-734016 & Ipilimumab & Yervoy & Fully humanized IgG1 antibody & BMS Medarex \\
\hline & CTLA-4 & MEDI1123 & Tremelimumab & Not approved & fully humanized IgG2 antibody & $\begin{array}{l}\text { Astrazeneca } \\
\text { MedImmune }\end{array}$ \\
\hline
\end{tabular}

B7 family molecules (CD80/B7-1 and CD86/B7-2) on antigen-presenting cells to CD28 on T cells, resulting in T-cell activation. This is called the priming phase. Following activation, these T cells travel through the bloodstream to the tumor site where they recognize the tumor antigen presented by major histocompatibility complex molecules on the tumor cell surface and initiate an immune response. This is called the effector phase. During this phase, activated cytotoxic T lymphocytes can directly kill tumor cells by releasing perforin and granzymes. This entire process has traditionally been called the cancer-immunity cycle [1].

\section{Ongoing Trials of Immune Checkpoint Inhibitors for HCC}

Ongoing trials for HCC are summarized in Tables 2 and 3.

\section{Nivolumab}

Promising results from a phase I/II trial of the anti-PD-1 antibody nivolumab (the CheckMate 040 trial) were published in The Lancet [2]. On the sole basis of these results, the United States Food and Drug Administration (FDA) designated nivolumab for priority review as a second-line therapy for HCC in March 2017 and granted accelerated approval in September 2017.

The dose escalation component of the trial demonstrated the safety of nivolumab at up to $3 \mathrm{mg} / \mathrm{kg}$ in patients with hepatitis C virus (HCV) or hepatitis B virus (HBV) infection, and at up to $10 \mathrm{mg} / \mathrm{kg}$ in uninfected patients. The trial, which was split into dose escalation and dose expansion arms, investigated the efficacy and safety of nivolumab in a total of 214 patients. Response to treatment, including three complete responses, was achieved in 42 patients $(20 \%)$, in a population in which 144 patients $(67 \%)$ had extrahepatic spread and 63 patients (29\%) had vascular invasion. The disease control rate was 64\% (138/214 patients). The 9-month survival rate was $74 \%$, which is a very promising outcome considering the unfavorable tumor characteristics of this patient population with advanced HCC.

The length and durability of treatment responses is particularly noteworthy. Responders achieved responses within 3-4 months, and the responses were durable and persisted for a long period of time (median, 9.9 months). A spider plot of the data shows that responses were durable not only for complete and partial responders but also for patients with stable disease. 
Kudo: Immuno-Oncology Therapy for Hepatocellular Carcinoma: Current Status and Ongoing Trials

Table 2. Randomized phase II, phase III clinical trials of early/intermediate stage HCC

\begin{tabular}{|c|c|c|c|c|c|c|c|}
\hline Target population & \multicolumn{2}{|c|}{ Design } & Trial name & Result & Presentation & Publication & First author \\
\hline \multicolumn{8}{|l|}{ Early } \\
\hline \multirow{6}{*}{$\begin{array}{l}\text { Adjuvant (prevention } \\
\text { of recurrence) }\end{array}$} & 1 & Vitamin K2 vs. placebo & & Negative & & Hepatology 2011 & Yoshida $\mathrm{H}$ \\
\hline & 2 & Peretinoin vs. placebo & NIK-333 & Negative & ASCO 2010 & JG 2014 & Okita K \\
\hline & 3 & Sorafenib vs. placebo & STORM & Negative & ASCO 2014 & Lancet-0 2015 & Bruix J \\
\hline & 4 & Peretinoin vs. placebo & NIK-333/K-333 & Negative & & & \\
\hline & 5 & Nivolumab vs. placebo & CheckMate 9DX & Ongoing & & & \\
\hline & 6 & $\begin{array}{l}\text { Durvalumab } \pm \\
\text { bevacizumab vs. placebo }\end{array}$ & EMERALD-2 & Ongoing & & & \\
\hline \multirow[t]{2}{*}{ Improvement of RFA } & 1 & RFA \pm LTLD & HEAT & Negative & ILCA 2013 & CCR 2017 & Tak WY \\
\hline & 2 & $\mathrm{RFA} \pm \mathrm{LTLD}$ & OPTIMA & & & & \\
\hline \multicolumn{8}{|l|}{ Intermediate } \\
\hline \multirow[t]{6}{*}{ Improvement of TACE } & 1 & $\mathrm{TACE} \pm$ sorafenib & Post-TACE & Negative & ASCO-GI 2010 & EJC 2011 & Kudo M \\
\hline & 2 & $\mathrm{TACE} \pm$ sorafenib & SPACE (Ph II) & Negative & ASCO-GI 2012 & J Hepatol 2016 & Lencioni R \\
\hline & 3 & $\mathrm{TACE} \pm$ brivanib & BRISK-TA & Negative & ILCA 2013 & Hepatol 2014 & Kudo M \\
\hline & 4 & $\mathrm{TACE} \pm$ orantinib & ORIENTAL & Negative & EASL 2015 & Lancet GH 2017 & Kudo M \\
\hline & 5 & TACE \pm sorafenib & TACE-2 & Negative & ASCO 2016 & Lancet GH 2017 & Meyer T \\
\hline & 6 & $\mathrm{TACE} \pm$ sorafenib & TACTICS (Ph II) & Positive & ASCO-GI 2018 & & Kudo M \\
\hline
\end{tabular}

Red, positive trial; blue, ongoing trials; black, negative trials; LTLD, lyso-thermosensitive liposomal doxorubicin.

Table 3. Phase III clinical trials in advanced HCC [21]

\begin{tabular}{|c|c|c|c|c|c|c|c|}
\hline Target & \multicolumn{2}{|c|}{ Design } & Trial name & Result & Presentation & Publication & First author \\
\hline \multirow{15}{*}{$\begin{array}{l}\text { Advanced } \\
1 \text { st line }\end{array}$} & & & & & & & \\
\hline & 1 & Sorafenib vs. sunitinib & SUN1170 & Negative & ASCO 2011 & JCO 2013 & Cheng AL \\
\hline & 2 & Sorafenib \pm erlotinib & SEARCH & Negative & ESMO 2012 & JCO 2015 & Zhu AX \\
\hline & 3 & Sorafenib vs. brivanib & BRISK-FL & Negative & AASLD 2012 & JCO 2013 & Johnson PJ \\
\hline & 4 & Sorafenib vs. linifanib & LiGHT & Negative & ASCO-GI 2013 & JCO 2015 & Cainap C \\
\hline & 5 & Sorafenib \pm doxorubicin & CALGB 80802 & Negative & ASCO-GI 2016 & & \\
\hline & 6 & Sorafenib \pm HAIC & SILIUS & Negative & EASL 2016 & Lancet GH 2018 & Kudo M \\
\hline & 7 & Sorafenib \pm Y 90 & SARAH & Negative & EASL 2017 & Lancet-0 2017 & Vilgrain V \\
\hline & 8 & Sorafenib \pm Y90 & SIRveNIB & Negative & ASCO 2017 & JCO 2018 & Chow P \\
\hline & 9 & Sorafenib vs. lenvatinib & REFLECT & Positive & ASCO 2017 & Lancet 2018 & Kudo M \\
\hline & 10 & Sorafenib vs. nivolumab & CheckMate-459 & Negative & & & \\
\hline & 11 & $\begin{array}{l}\text { Sorafenib vs. durvalumab + tremelimumab } \\
\text { vs. durva }\end{array}$ & HIMALAYA & Ongoing & & & \\
\hline & 12 & Sorafenib vs. atezolizumab + bevacizumab & IMbrave150 & Ongoing & & & \\
\hline & 13 & Sorafenib vs. tislelizumab & Rationale-301 & Ongoing & & & \\
\hline & 14 & Lenvatinib vs. lenvatinib + pembrolizumab & LEAP 002 & Ongoing & & & \\
\hline \multirow[t]{12}{*}{ 2nd line } & 1 & Brivanib vs. placebo & BRISK-PS & Negative & EASL 2012 & JCO 2013 & Llovet JM \\
\hline & 2 & Everolimus vs. placebo & EVOLVE-1 & Negative & ASCO-GI 2014 & JAMA 2014 & Zhu AX \\
\hline & 3 & Ramucirumab vs. placebo & REACH & Negative & ESMO 2014 & Lancet-0 2015 & Zhu AX \\
\hline & 4 & S-1 vs. placebo & S-CUBE & Negative & ASCO 2015 & Lancet GH 2017 & Kudo M \\
\hline & 5 & ADI-PEG 20 vs. placebo & NA & Negative & ASCO 2016 & Ann Oncol 2018 & Abou-Alfa GK \\
\hline & 6 & Regorafenib vs. placebo & RESORCE & Positive & WCGC 2016 & Lancet 2017 & Bruix J \\
\hline & 7 & Tivantinib vs. placebo & METIV-HCC & Negative & ASCO 2017 & Lancet-0 2018 & Rimassa L \\
\hline & 8 & Tivantinib vs. placebo & JET-HCC & Negative & ESMO 2017 & & \\
\hline & 9 & DT vs. placebo & ReLive & Negative & ILCA 2017 & & \\
\hline & 10 & Cabozantinib vs. placebo & CELESTIAL & Positive & ASCO-GI 2018 & NEJM 2018 & Abou-Alfa GK \\
\hline & 11 & Ramucirumab vs. placebo & REACH-2 & Positive & ASCO 2018 & Lancet 2018 & Zhu AX \\
\hline & 12 & Pembrolizumab vs. placebo & KEYNOTE-240 & Negative & ASCO 2019 & & \\
\hline
\end{tabular}

Black, negative; red, positive; blue, ongoing; HAIC, hepatic arterial infusion chemotherapy; DT, doxorubicin-loaded nanoparticles.

However, $30-40 \%$ of patients did not respond at all, and disease progressed quickly in some of these patients. This could be a limitation of nivolumab monotherapy.

In summary, treatment of HCC with nivolumab monotherapy yielded similarly durable responses as have been seen in other types of cancer. This is characteristic of immune checkpoint inhibitors and merits attention. Evaluation of adverse reactions showed a very low incidence of grade 3-4 increased aspartate aminotransferase levels ( 9 patients, 4\%) and increased 


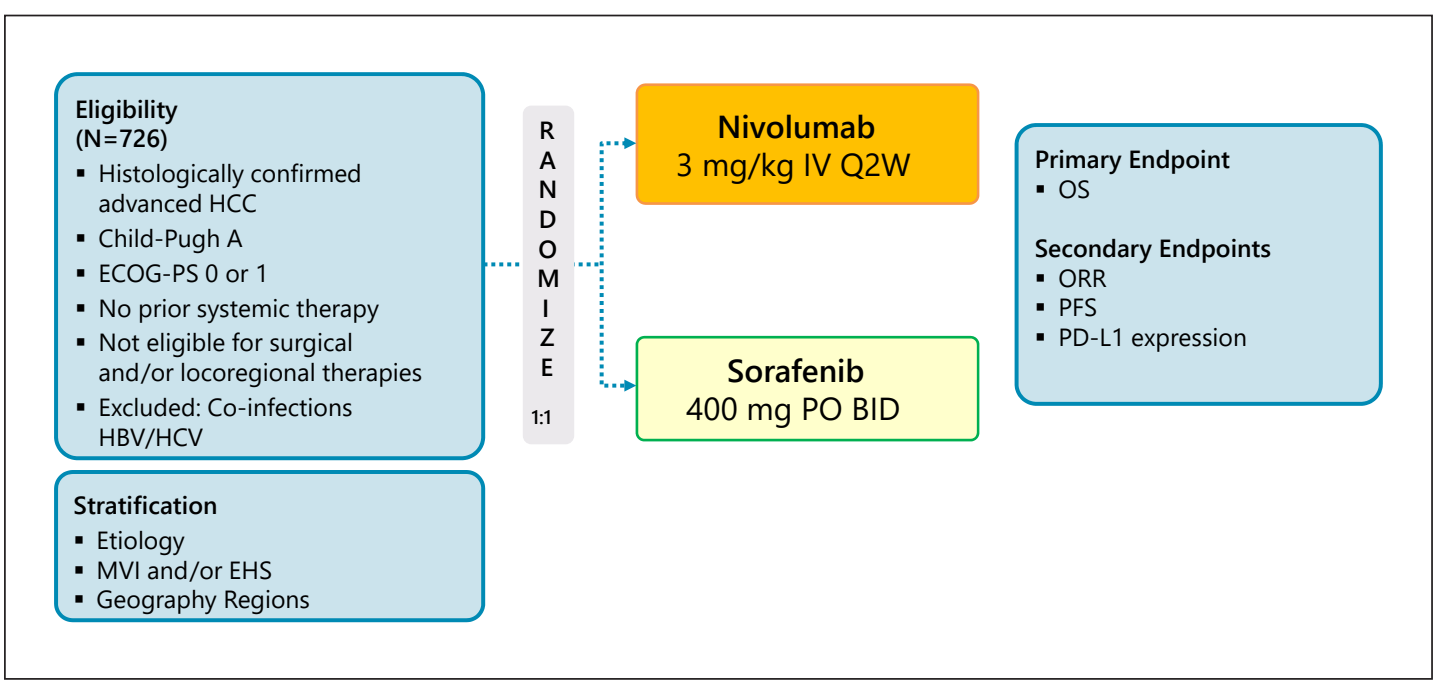

Fig. 1. CheckMate 459: nivolumab in first line. Clinical trial information: NCT02576509 [33].

alanine aminotransferase levels ( 6 patients, $3 \%$ ), which were the most concerning treatmentrelated adverse reactions. These results demonstrated that nivolumab resulted in comparable hepatic impairment in HCC as in other cancers. Though serious hepatic impairment associated with viral hepatitis had been a concern, not one case was reported.

The results of this trial can be summarized in three points, as follows: First, monotherapy with the anti-PD-1 antibody nivolumab showed a sufficiently favorable safety profile in HCC, similar to the safety profile observed in other types of cancer. Second, nivolumab can be used safely in patients infected with HBV or HCV. Third, immunotherapy yielded a relatively high response rate, and the responses were durable. This durability was observed at all dose levels and in all cohorts (uninfected, HBV-infected, and HCV-infected patients).

When follow-up data were reported at the 2017 annual meeting of the American Society of Clinical Oncology (ASCO), overall survival (OS) rates were remarkably favorable, at 28.6 months in sorafenib-naïve patients and 15.6 months in sorafenib-experienced patients.

A head-to-head phase III trial of nivolumab and sorafenib (the CheckMate 459 trial) has been performed (Table 3; Fig. 1); however, the press release by Bristol-Myers Squibb on June 24, 2019 announced results from CheckMate-459 study nivolumab as a first-line treatment for patients with unresectable HCC were negative. The trial did not achieve statistical significance for its primary endpoint of overall survival per the pre-specified analysis (HR $=0.85$ [95\% CI: 0.72-1.02]; $p=0.0752$ ). While CheckMate-459 did not reach its pre-specified primary endpoint, the results showed a trend towards improvement in OS for patients treated with nivolumab compared to sorafenib (https://news.bms.com).

\section{Pembrolizumab}

Phase II KEYNOTE-224 Trial

Pembrolizumab is a potent, highly selective humanized IgG4/ $/$ monoclonal antibody that directly inhibits the binding of PD-1 to its ligands, PD-L1 and PD-L2. It has already been approved for the treatment of melanoma, non-small cell lung cancer, squamous cell carcinoma, gastric cancer, urothelial cancer, and classical Hodgkin's lymphoma [3, 4]. Pembrolizumab was also granted accelerated approval by the FDA on November 10, 2018, as a second-line agent for HCC to be used after treatment with sorafenib, based on the results of the phase II KEYNOTE-224 trial [5]. 


\section{Liver

Kudo: Immuno-Oncology Therapy for Hepatocellular Carcinoma: Current Status and Ongoing Trials

The KEYNOTE-224 trial was a non-randomized, multicenter, open-label, phase II trial. There were 47 participating institutions from 10 countries, and 104 patients were enrolled from June 7, 2016, to February 9, 2017. The main inclusion criteria were (1) a histopathologically confirmed diagnosis, (2) documented radiographic progression after stopping treatment with sorafenib or intolerance to sorafenib, (3) an Eastern Cooperative Oncology Group (ECOG) performance status of 0-1, (4) a Child-Pugh classification of A, and (5) adequate organ function.

Efficacy results from the trial were as follows. As of the data cut-off date of February 13, 2018, a total of 17 patients (16\%) were still being treated with pembrolizumab. The median duration of follow-up was 12.3 months (interquartile range, 7.6-15.1). Of the 104 patients, 18 responded, and the response rate was $17 \%$ (95\% confidence interval [CI], 11-26). Fortysix patients (44\%) had stable disease, and 34 (33\%) had progressive disease (PD). The median time to response was 2.1 months (interquartile range, 2.1-4.1). Of the 18 responders, 12 $(67 \%)$ had achieved that response by the time of the initial response assessment by imaging evaluation, which was performed between weeks 8 and 10. The median duration of response was not reached (3.1-14.6+), but the duration of response was 9 months or longer in 12 patients $(77 \%)$.

At the data cut-off date, death or PD was reported for 84 patients $(81 \%$; 60 deaths and 24 patients with PD). The median progression-free survival (PFS) was 4.9 months (95\% CI, 3.9-8.0), and the median OS was 12.9 months (95\% CI, 9.7-15.5) [5].

Safety results from the trial were as follows. At least one adverse event (AE) was observed in 101 patients (97\%). Treatment-related adverse events (TRAEs) were observed in 76 patients (73\%), and serious TRAEs were observed in 16 of these patients (15\%). Grade 3 TRAEs, specifically increased aspartate aminotransferase (7 patients, $7 \%$ ) and fatigue (4 patients, $4 \%$ ), were observed in 25 patients (24\%). The only grade 4 TRAE was hyperbilirubinemia, which was observed in 1 patient (1\%). There was also 1 treatment-related death from ulcerative esophagitis. Immune-mediated AEs were observed in 15 patients $(14 \%)$. These included hypothyroidism (8\%) and adrenal insufficiency (3\%). Grade 3 immunemediated AEs were adrenal insufficiency ( 2 patients), severe skin toxicity ( 1 patient), and type 1 diabetes mellitus ( 1 patient). Immune-mediated hepatitis was observed in 3 patients (3\%), but hepatitis B or C flares were not observed.

PD-L1 expression is an established predictor of response to the anti-PD-1 antibody pembrolizumab in other solid tumors, particularly non-small cell lung cancer and gastric cancer. Immune cell infiltration is related to clinical efficacy in HCC as well [6].

In this trial, tissue samples for pathological evaluation were obtained from 52 of the 104 enrolled patients. Of these, 47 were archival samples and the other 5 were newly obtained biopsy samples. There was no significant difference in patient characteristics between this subgroup of 52 patients and the overall patient population. In particular, the lack of difference in critical prognostic factors such as alpha-fetoprotein levels, Barcelona Clinic Liver Cancer stage, ECOG performance status, extrahepatic spread, and macrovascular invasion makes this group a truly unbiased, representative sample of all enrolled patients. The objective response rate (ORR) differed very little between these 52 patients (13 of 52, 25\%) and the overall patient population (17\%).

The relationship between PD-L1 expression and response was evaluated. Two new indices of PD-L1 expression were used in this trial. The first was the combined positive score (CPS), which was defined as the number of PD-L1-positive cells (tumor cells, lymphocytes, and macrophages) divided by the total number of viable tumor cells and multiplied by 100 . The second was the tumor proportion score (TPS), which was defined as the number of tumor cells that express PD-L1 (either partial or complete staining) divided by the total number of viable tumor cells and multiplied by 100 . Twenty-two of the 52 patients (42\%) were CPS 
Kudo: Immuno-Oncology Therapy for Hepatocellular Carcinoma: Current Status and Ongoing Trials

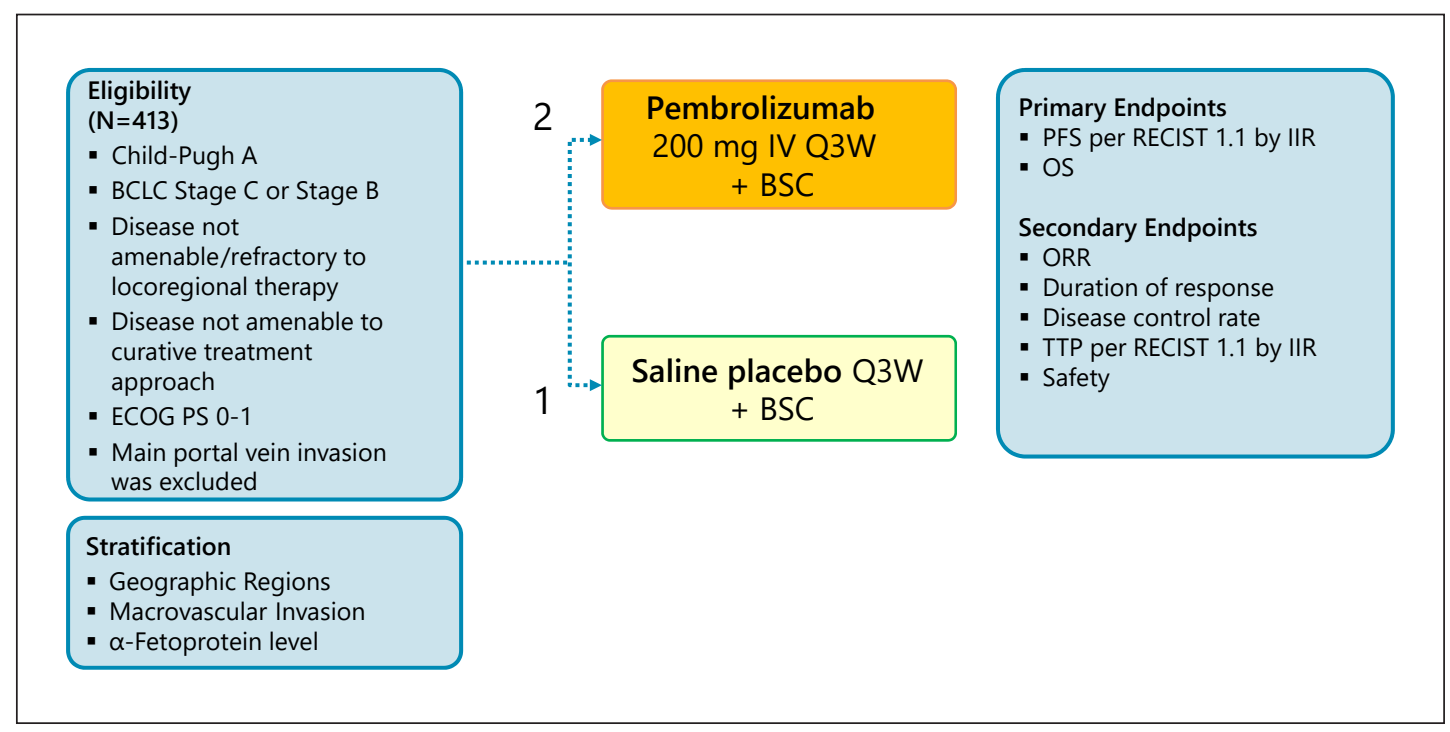

Fig. 2. KEYNOTE-240: pembrolizumab in second line. Clinical trial information: NCT02702401 [34].

positive, and 7 of $52(13 \%)$ were TPS positive. An objective response (OR) was observed in 13 patients $(25 \%)$. ORs were observed in significantly fewer CPS-negative patients ( 6 of 30 , $20 \%$ ) than CPS-positive patients (7 of 22,32\%) ( $p=0.021)$. With respect to the TPS, ORs were observed in 3 of 7 TPS-positive patients (43\%) and 10 of 45 TPS-negative patients (22\%). Perhaps due to the small number of patients, the different in ORR between TPS-positive and TPS-negative patients was not significant $(p=0.088)$ [5].

The same results were obtained for PFS; there was a significant difference for CPS ( $p=$ $0.026)$ but not TPS ( $p=0.096)$. These results are consistent with those for nivolumab and may also reflect the tumor heterogeneity in biopsy sampling for HCC [2].

In light of these results, the FDA approved pembrolizumab as a second-line agent for HCC in patients intolerant of or refractory to sorafenib.

Phase III KEYNOTE 240 Trial

The results of the KEYNOTE-240 trial were presented at ASCO 2019 (Fig. 2).

A total of 413 patients with BCLC B or C stage HCC were randomized at a 2:1 ratio to treatment with pembrolizumab $(n=278)$ or placebo $(n=135)$. Stratification factors included geographic region (Asia, excluding Japan vs. non-Asia, plus Japan), macrovascular invasion (yes vs. no), and alpha-fetoprotein level ( $\geq 200$ vs. $<200 \mathrm{ng} / \mathrm{mL}$ ). Patients with main portal vein invasion were excluded (Fig. 2).

Regarding post-study anticancer therapy, anti-PD-1/PD-L1 agents were given to 14 patients $(10.4 \%)$ in the placebo arm and to 19 patients $(6.8 \%)$ in the pembrolizumab arm. Other active agents, such as regorafenib, lenvatinib, cabozantinib, and ramucirumab, were given to 69 patients (24.8\%) in the pembrolizumab arm and to 29 patients $(21.5 \%)$ in the placebo arm. The safety profile, including the incidence of immune-related AEs and hepatitis, was similar to that of pembrolizumab in other cancer types; no HBV or HCV flares were identified [7].

In this trial, the co-primary endpoints were PFS and OS, and interim analyses were performed twice. Therefore, the one-sided type I error $(\alpha)$ was determined using a splitsample analysis and OS testing was performed by group sequential design, as follows: PFS, $\alpha=0.002$; OS, $\alpha=0.023$; OS at the first interim analysis, $\alpha=0.0064$; OS at the second interim analysis, $\alpha=0.0129$; and OS at the final analysis, $\alpha=0.0174$ [7]. 


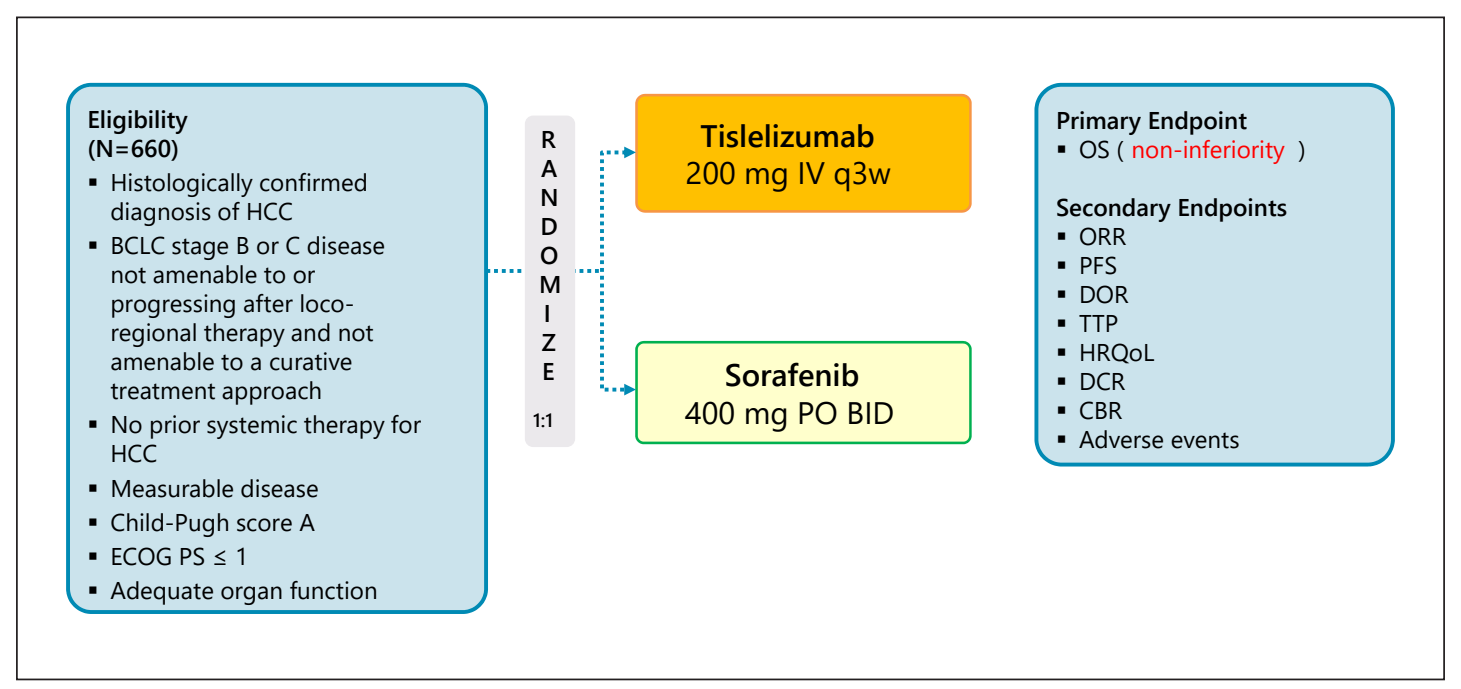

Fig. 3. RATIONALE-301: tislelizumab in first line (non-inferiority trial). Clinical trial information: NCT03412773 [35].

Table 4. Phase III trial in second-line setting

\begin{tabular}{|c|c|c|c|c|c|c|c|}
\hline \multirow[t]{2}{*}{ Trials } & \multirow{2}{*}{$\begin{array}{l}\text { Primary } \\
\text { endpoint }\end{array}$} & \multirow[t]{2}{*}{ Results } & \multirow[t]{2}{*}{$n$} & \multicolumn{2}{|l|}{ os } & \multicolumn{2}{|c|}{ PFS or TTP } \\
\hline & & & & $\overline{\mathrm{HR}}$ & $95 \% \mathrm{CI}$ & $\overline{\mathrm{HR}}$ & $95 \% \mathrm{CI}$ \\
\hline BRISK-PS [40] & os & Negative & 395 & 0.89 & $0.69-1.15$ & 0.56 & $0.42-0.76$ \\
\hline EVOLVE-1 [41] & os & Negative & 546 & 1.05 & $0.86-1.27$ & 0.93 & $0.75-1.15$ \\
\hline S-CUBE [42] & OS & Negative & 399 & 0.86 & $0.67-1.10$ & 0.60 & $0.46-0.77$ \\
\hline REACH [43] & os & Negative & 544 & 0.87 & $0.72-1.05$ & 0.59 & $0.49-0.72$ \\
\hline REACH-2 [44] & OS & Positive & 292 & 0.71 & $0.531-0.949$ & 0.452 & $0.339-0.603$ \\
\hline RESORSE [45] & OS & Positive & 573 & 0.63 & $0.50-0.79$ & 0.46 & $0.37-0.56$ \\
\hline CELESTIAL [46] & OS & Positive & 707 & 0.76 & $0.63-0.92$ & 0.44 & $0.36-0.52$ \\
\hline Keynote-240 [34] & OS/PFS & Negative & 408 & 0.78 & $\begin{array}{l}0.611-0.998 \\
p=0.0238\end{array}$ & 0.78 & $\begin{array}{c}0.61-0.99 \\
p=0.0209\end{array}$ \\
\hline
\end{tabular}

In the final analysis, the median OS was 13.9 months in the pembrolizumab arm, compared with 10.6 months in the placebo arm (HR, $0.781 ; 95 \% \mathrm{CI}, 0.611-0.998 ; p=0.0238)$. The final median PFS in the pembrolizumab arm was 3.0 months (95\% CI, 2.8-4.1), compared with 2.8 months (95\% CI, 1.6-3.0) in the placebo arm (HR, 0.718; 95\% CI, 0.570-0.904; $p=0.0022$ ). Both PFS and OS failed to reach the prespecified statistical significance ( $p=0.002$ for PFS and $p=0.0174$ for OS). The ORR was $18.3 \%$ (95\% CI, 14.0-23.4) in the pembrolizumab arm and $4.4 \%$ (95\% CI, 1.6-9.4) in the placebo arm ( $p=0.00007$; odds ratio, 13.8 [7.7-19.9]) [7].

These results showed an apparent survival benefit of pembrolizumab over the placebo; however, this study unfortunately did not meet its primary endpoint of prolonging OS or PFS per prespecified statistical plan. As shown in Table 4, the HR for OS in the pembrolizumab arm was comparable with that of other agents in the positive trials.

\section{Tislelizumab}

Tislelizumab is a humanized IgG4 antibody to PD-1, and a phase III trial of this antibody is ongoing. This trial follows a non-inferiority design (Non-inferiority margin, 1.08), unlike the other trials (Table 3; Fig. 3). 
Kudo: Immuno-Oncology Therapy for Hepatocellular Carcinoma: Current Status and Ongoing Trials

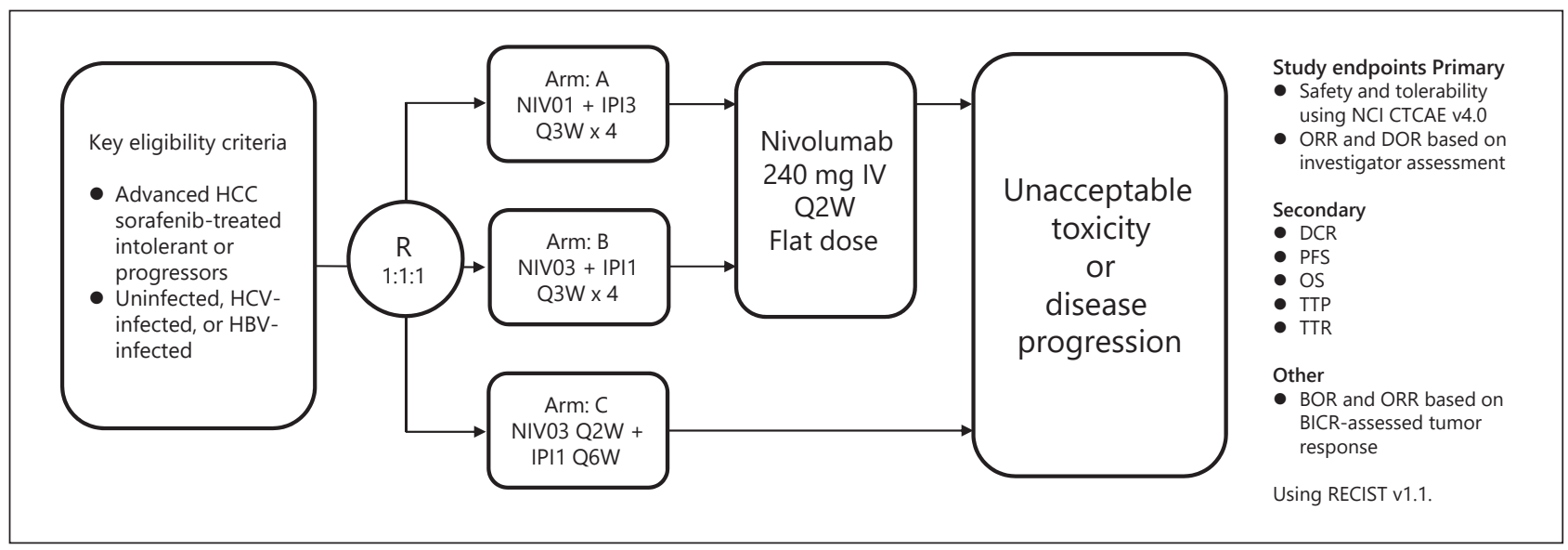

Fig. 4. CheckMate 040 nivolumab plus ipilimumab combination cohort study design. Minimum follow-up at time of data cutoff: 28 months. BICR, blinded independent central review; BOR, best overall response; DOR, duration of response; IPI1, ipilimumab $1 \mathrm{mg} / \mathrm{kg}$; IPI3, ipilimumab $3 \mathrm{mg} / \mathrm{kg}$; NCI CTCAE, National Cancer Institute Common Terminology Criteria for Adverse Events; NIV01, nivolumab $1 \mathrm{mg} / \mathrm{kg}$; NIV03, nivolumab $3 \mathrm{mg} / \mathrm{kg}$; PFS, progression-free survival; R, randomization; TTP, time to progression; TTR, time to response.

\section{Combination Immunotherapy with other Treatment Modalities}

\section{Combination Therapy with a PD-1/PD-L1 Antibody and a CTLA-4 Antibody}

Rationale

Antibodies against PD-1, PD-L1, and CTLA-4 have all shown promise in HCC, and combination therapy for simultaneous inhibition of several immune checkpoints is another approach to immunotherapy that is currently being investigated in clinical trials. The observation that the combination of nivolumab plus ipilimumab yielded better treatment outcomes than ipilimumab monotherapy in melanoma $[8,9]$ has inspired the use of the same combination therapy for HCC $[10,11]$. The theoretical premise for combination of anti-PD-1/PD-L1 antibodies with anti-CTLA-4 antibodies is that if there are no CD8-positive T cells in the tumor tissue, inhibition of the PD-1/PD-L2 pathway alone will not activate antitumor immunity when crucial lymphocytes are not present locally in the target area. However, concurrent inhibition of the B7-CTLA-4 pathway with anti-CTLA-4 antibodies increases the number of activated CD8-positive cells in the lymph nodes, as well as the number of tumor-infiltrating activated CD8-positive T cells, thereby enhancing the antitumor effect of anti-PD-1/PD-L1 antibodies. In addition, regulatory $\mathrm{T}$ cells (Tregs) that infiltrate tumors and downregulate tumor immunity by expressing CTLA-4 on their surface [12], and thus inhibition of CTLA-4 on Tregs could be an additional mechanism through which anti-CTLA-4 antibodies increase the antitumor activity of CD8-positive T cells. This hypothesis inspired the initiation of several ongoing clinical trials investigating the combination of anti-CTLA-4 and anti-PD-1/PD-L1 antibodies for HCC.

Nivolumab plus Ipilimumab (CheckMate 040 Cohort 4)

The CheckMate 040 trial (cohort 4) is investigating the combination of nivolumab and the anti-CTLA-4 antibody ipilimumab at various doses and dose intervals (Table 3; Fig. 4).

A total of 148 patients with advanced HCC after sorafenib treatment were randomized 1:1:1 to Arms A, B, and C (Fig. 4). The Arm A regimen consisted of four cycles of nivolumab at $1 \mathrm{mg} / \mathrm{kg}$ plus ipilimumab at $3 \mathrm{mg} / \mathrm{kg}$ every 3 weeks, followed by a $240-\mathrm{mg}$ flat dose of 
Kudo: Immuno-Oncology Therapy for Hepatocellular Carcinoma: Current Status and Ongoing Trials

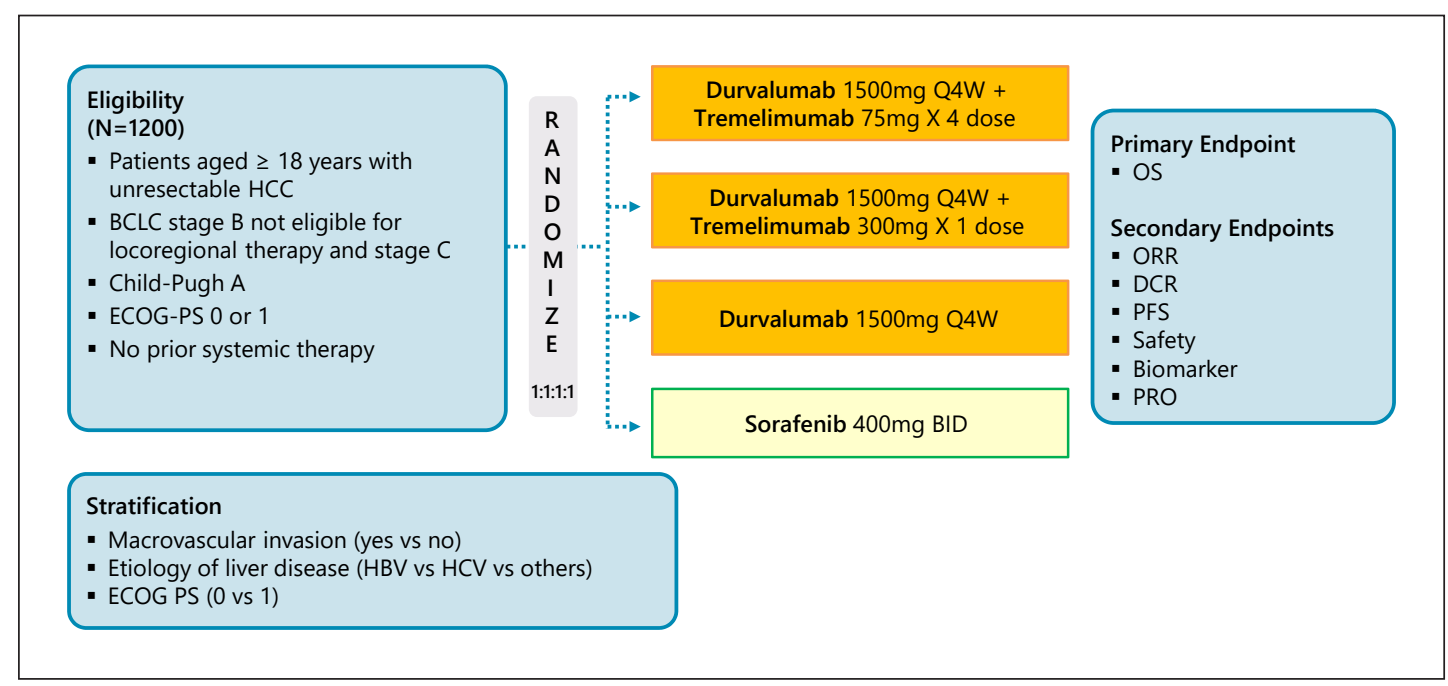

Fig. 5. HIMALAYA: durvalumab + tremelimumab in first line. Clinical trial information: NCT03298451 [36].

nivolumab every 2 weeks. The Arm B regimen consisted of nivolumab at $3 \mathrm{mg} / \mathrm{kg}$ plus ipilimumab at $1 \mathrm{mg} / \mathrm{kg}$, followed by a $240-\mathrm{mg}$ flat dose of nivolumab every 2 weeks. The Arm C regimen consisted of nivolumab at $3 \mathrm{mg} / \mathrm{kg}$ every 2 weeks plus ipilimumab every 6 weeks. The primary endpoints were safety and tolerability, and ORR and duration of response were determined based on Response Evaluation Criteria in Solid Tumors (RECIST) 1.1. The ORR was 32\% (16/50) in Arm A, 31\% (15/49) in Arm B, and 31\% (15/49) in Arm C. A complete response was seen in $8 \%$ of patients $(n=4)$ in Arm A and $6 \%$ of patients $(n=3)$ in Arm B. The median OS in Arms A, B, and C was 22.8 months (9.4-not evaluable), 12.5 months (7.6-16.4), and 12.7 months (7.4-33.0), respectively. The ORR was similar between patients with PD-L1 expression $<1$ and $>1 \%$ [13].

The Arm A regimen seems to show the best survival benefit, with an acceptable safety profile [13].

Phase III HIMALAYA Trial (Durvalumab plus Tremelimumab)

Another phase I/II trial is investigating the efficacy and safety of combination therapy with the anti-PD-L1 antibody durvalumab and the anti-CTLA-4 antibody tremelimumab. According to phase I results from the trial presented at ASCO 2017, the response rate among 40 patients with HCC treated with durvalumab plus tremelimumab was $25.0 \%$, which was better than that reported for patients treated with durvalumab alone [10]. These results led to the initiation of a phase III trial (the HIMALAYA trial), which is currently underway. It started as a four-arm study but was changed to a three-arm study with protocol amendments in October 2018. Essentially, it is a comparative trial of durvalumab plus tremelimumab, durvalumab alone, and sorafenib (Fig. 5).

\section{Combination Immunotherapy with Locoregional Therapies}

Another approach being investigated is the combination of immune checkpoint inhibitors with existing locoregional therapies for HCC. Transarterial chemoembolization (TACE), radiofrequency ablation (RFA), and radiotherapy can enhance the effects of immunotherapy because they activate the immune system by inducing local inflammation, which triggers release of neoantigens. These locoregional therapies would presumably be particularly beneficial when antigen release is poor and cancer antigens are not being released. Results from 
Kudo: Immuno-Oncology Therapy for Hepatocellular Carcinoma: Current Status and Ongoing Trials

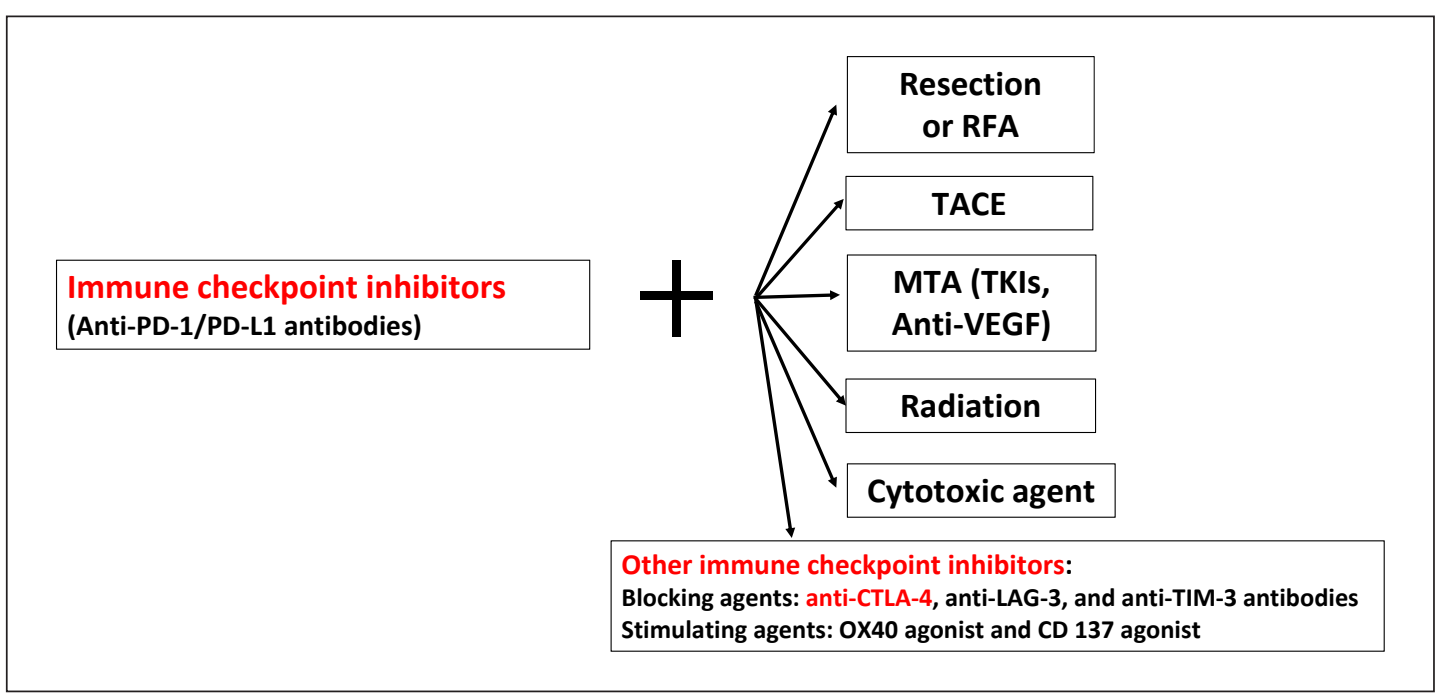

Fig. 6. Combination Immunotherapy in hepatocellular carcinoma. RFA, radiofrequency ablation; TACE, transarterial chemoembolization; TKIs, tyrosine kinase inhibitors.

Table 5. Clinical trials of IO as adjuvant or neoadjuvant after resection/RFA

\begin{tabular}{lllll}
\hline Phase & Target & Agent & Company & Trial No. \\
\hline 3 & PD-1 & Nivolumab (adjuvant, name: CheckMate 9DX) & BMS & NCT03383458 \\
\hline 3 & PD-L1 \pm VEGFR2 & Duravalumab \pm bevacizumab (adjuvant, name: EMERALD-2) & AstraZeneca & NCT03847428 \\
\hline 2 & PD-1 & Nivolumab (adjuvant, name: NIVOLVE) & Kindai Univ. (JP) & UMIN000026648 \\
\hline 2 & PD-1 + CTLA-4 & Nivolumab + ipilimumab (neoadjuvant + adjuvant) & MD Anderson CC & NCT03222076 \\
\hline 1 & PD-1 + MET/VEGFR2 & Nivolumab + cabozantinib (neoadjuvant) & Sidnet Kimmel C-CC NCT03299464 \\
\hline
\end{tabular}

a trial that investigated the combination of locoregional therapy with an anti-CTLA-4 inhibitor for advanced HCC were published in 2016 [14]. This was an adjuvant trial in which patients with advanced HCC were treated with the anti-CTLA-4 antibody tremelimumab after undergoing RFA or TACE targeted only at certain nodular lesions. The results were favorable, with a partial response rate of $26 \%$, a time to progression of 7.4 months, and an OS of 12.3 months. Increases in CD3- and CD8-positive cells were even observed in untreated nodules. This phenomenon is referred to as the abscopal effect.

Immune checkpoint inhibitors (anti-PD-1, anti-PD-L1, and anti-CTLA-4 antibodies) may be beneficial in a variety of settings, including as neoadjuvant therapy, adjuvant therapy after resection or ablation, or in combination with TACE (Fig. 6).

This is particularly significant for HCC due to its high recurrence rate even after curative therapies such as resection or RFA. Tiny microsatellite lesions typically undetectable on imaging are the main cause of recurrence after curative therapy. Clinical trials have investigated the efficacy of various adjuvant therapies (e.g., interferons, retinoids, vitamin $\mathrm{K}$, and sorafenib) to address this problem, but these approaches have all failed (Table 2) [15-18].

However, when release of tumor antigen is induced by TACE or RFA, subsequent administration of an anti-PD-1 antibody may inhibit intrahepatic micrometastases [19]. Mizukoshi et al. [20] reported significant increases in tumor-specific $\mathrm{T}$ cells and tumor antigen release in $62 \%$ of patients after RFA. The authors also found that recurrence-free survival increased significantly with the increase in generation of tumor-specific T cells [20]. 
Kudo: Immuno-Oncology Therapy for Hepatocellular Carcinoma: Current Status and Ongoing Trials

Eligibility

$(\mathrm{N}=530)$

- Patients aged $\geq 18$ years with a first diagnosis of HCC (any etiology)

- Curative resection or complete ablation

- High risk of recurrence

- Child-Pugh score 5 or 6

- ECOG-PS $\leq 1$

- No microvascular invasion or metastatic disease

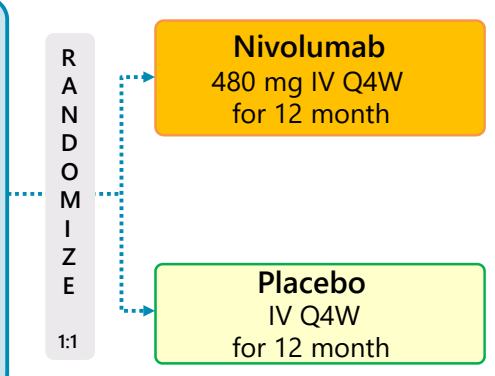

Primary Endpoint

- RFS

Secondary Endpoints

- OS

- TTR

- Safety and tolerability

- Biomarkers

- Pharmacokinetics

- Cancer-related quality of

life

Fig. 7. CheckMate 9DX: adjuvant therapy of nivolumab. Clinical trial information: NCT03383458 [37].

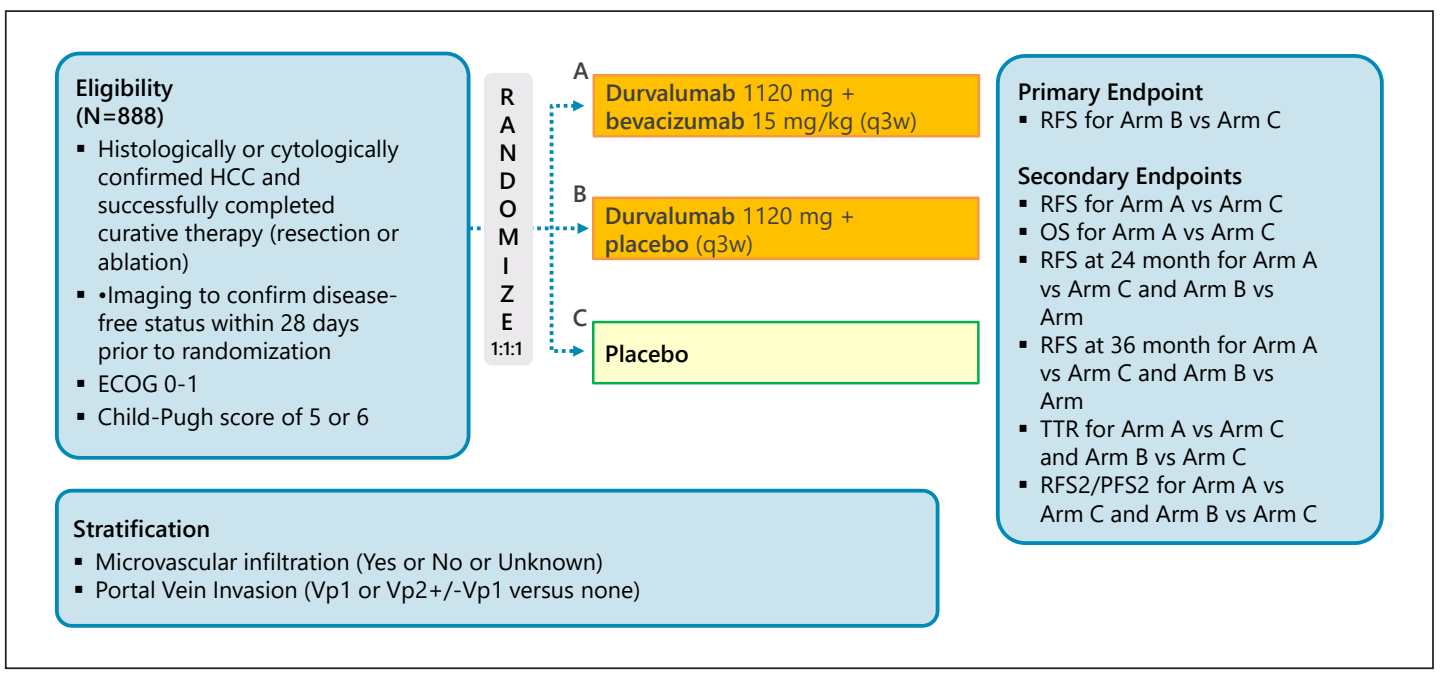

Fig. 8. EMERALD-2: adjuvant therapy of IO \pm VEGF-Ab. Clinical trial information: NCT03847428.

A phase III trial comparing recurrence-free survival between nivolumab and placebo after curative therapy (the CheckMate 9DX trial) is currently in progress (Table 5; Fig. 7). Another phase III trial, the EMERALD-2 trial (durvalumab \pm bevacizumab vs. placebo after curative therapy), is also ongoing (Fig. 8). A phase III trial of combination therapy with TACE and durvalumab and/or bevacizumab (the EMERALD-1 trial) is also underway (Fig. 9).

\section{Combination Immunotherapy with Molecular Targeted Agents}

Combinations of immune checkpoint inhibitors and molecular targeted agents have shown great promise in recent years. Vascular endothelial growth factor (VEGF)-A released by tumor cells and vascular endothelial cells at the tumor site activates and recruits numerous types of inhibitory immune cells, including tumor-associated macrophages, Tregs, and myeloid-derived suppressor cells, creating an immune-inhibitory microenvironment that favors tumor immune escape [21, 22]. These cells in turn release inhibitory cytokines (e.g., IL-10 and TGF- $\beta$ ) that impede dendritic cell maturation and inhibit NK and T cell activation and proliferation [22]. In the liver, stromal cells (e.g., Kupffer cells, dendritic cells, hepatic endothelial cells, and stellate cells) are believed to contribute to an immune-suppresive microenvironment [23]. Thus, inhibition of VEGF with an anti-VEGF antibody or a multi- 
Kudo: Immuno-Oncology Therapy for Hepatocellular Carcinoma: Current Status and Ongoing Trials

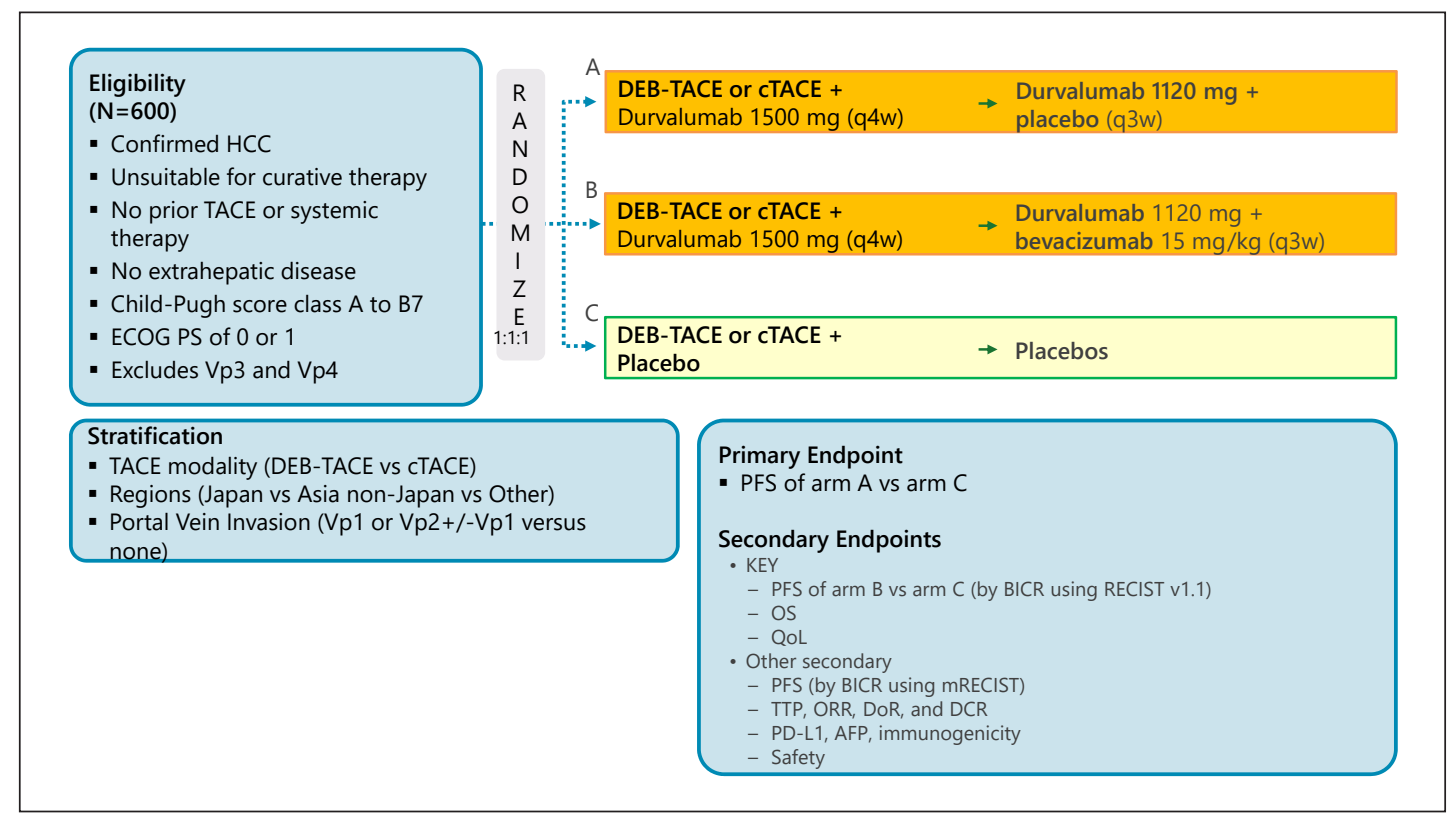

Fig. 9. EMERALD-1: TACE in combination with IO \pm VEGF-Ab. Clinical trial information: NCT03778957.

Table 6. Immune checkpoint inhibitors in combination with MTAs in HCC

\begin{tabular}{lllll}
\hline Phase & Target & Agent & Company & Trial No. \\
\hline $1-2$ & PD-1 + TGF $\beta$ receptor I & Nivolumab + galunisertib & Eli Lilly & NCT02423343 \\
1 & PD-L1 + VEGFR-2 & Durvalumab + ramucirumab & Eli Lilly & NCT02572687 \\
1 & PD-1 + multikinase & Pembrolizumab + nintedanib & Gustave Roussy & NCT02856425 \\
1 & PD-1 + multikinase & PDR001 + sorafenib & Novartis & NCT02988440 \\
$1-2$ & PD-1 + c-Met & PDR001 + capmatinib (INC280) & NCT02795429 \\
$1-2$ & PD-1; CTLA-4 + MET/VEGFR2 & Nivolumab + ipilimumab + cabozantinib & BMS & NCT03418922 \\
1 & PD-1 + multikinase & Nivolumab + lenvatinib & Pfizer & NCT03289533 \\
$1-2$ & PD-L1 + multikinase & Averumab + axitinib & Jiangsu HengRui Medicine & NCT02942329 \\
1 & PD-1 + multikinase & Camrelizumab + apatinib & Eisai & RCT03006926 \\
1 & PD-1 + multikinase & Pembrolizumab + lenvatinib & RCT03170960 \\
$1-2$ & PD-L1 + anti-VEGF & Atezolizumab + bevacizumab & NCT03434379 \\
3 & PD-L1 + anti-VEGF & Atezolizumab + bevacizumab & & \\
\hline
\end{tabular}

kinase inhibitor whose targets include VEGF could lead to (1) improved antigen presentation by dendritic cells, (2) increased T-cell priming, and (3) improved trafficking from the lymph nodes to the tumor site through normalization of the tumor vasculature. This would also result in (4) regulation of the humoral factors TGF- $\beta$ and IL-10 by inhibition of Tregs, tumorassociated macrophages, and myeloid-derived suppressor cells at the tumor site, promoting an inflammatory, rather than an inhibitory, immune microenvironment in the tumor. This would ultimately (5) enable activated $\mathrm{T}$ cells to effectively attack the tumor and induce necrosis [21]. In fact, nonclinical studies of combination therapy with pembrolizumab and lenvatinib have demonstrated that exclusion of tumor-associated macrophages and Tregs from the tumor microenvironment results in decreased production of TGF- $\beta$ and IL-10, as well as decreased expression of PD-1 and TIM-3. This suggests that proinflammatory cytokines such as IL-12 could induce antitumor immunity [24].

Several clinical trials investigating the combination of immune checkpoint inhibitors and molecular targeted therapies have been initiated to test this hypothesis (Table 6). As reported at the European Society for Medical Oncology (ESMO) congress in 2016 and the ASCO annual 


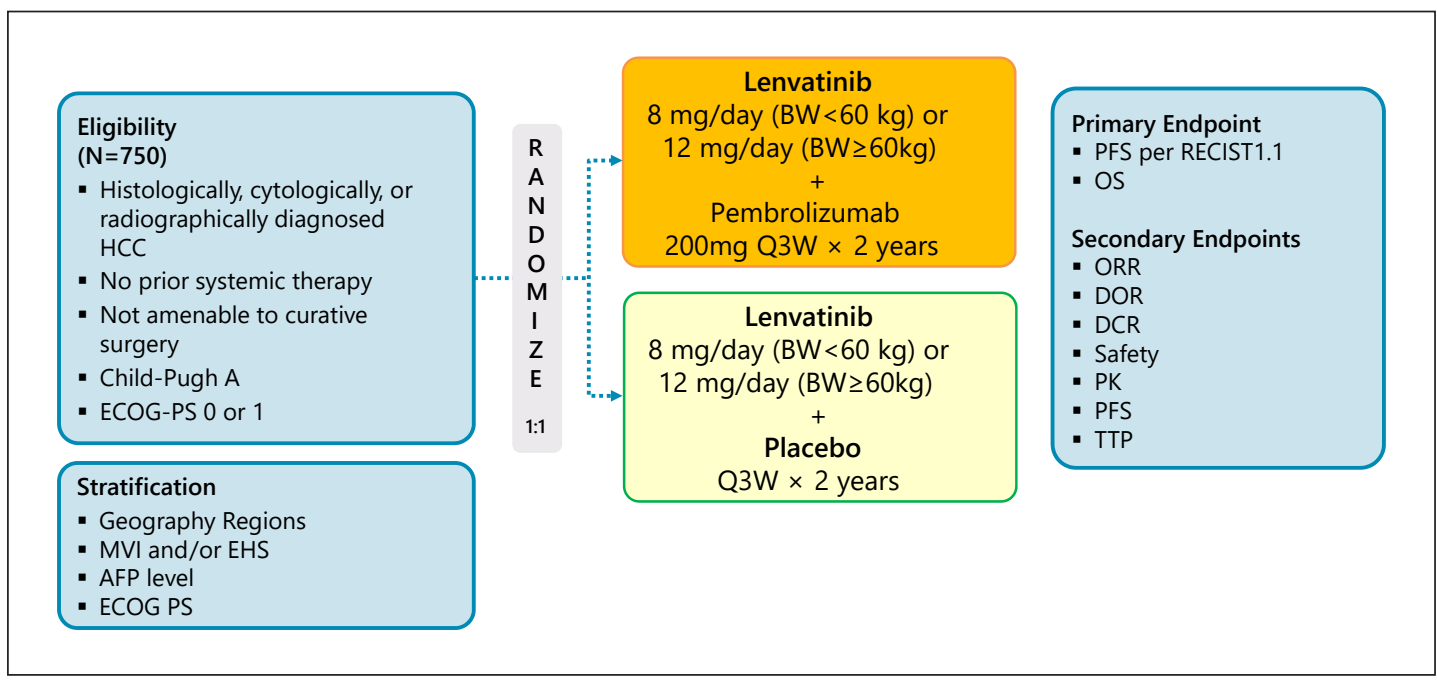

Fig. 10. LEAP-002: lenvatinib + pembrolizumab in first line. Clinical trial information: NCT03713593.

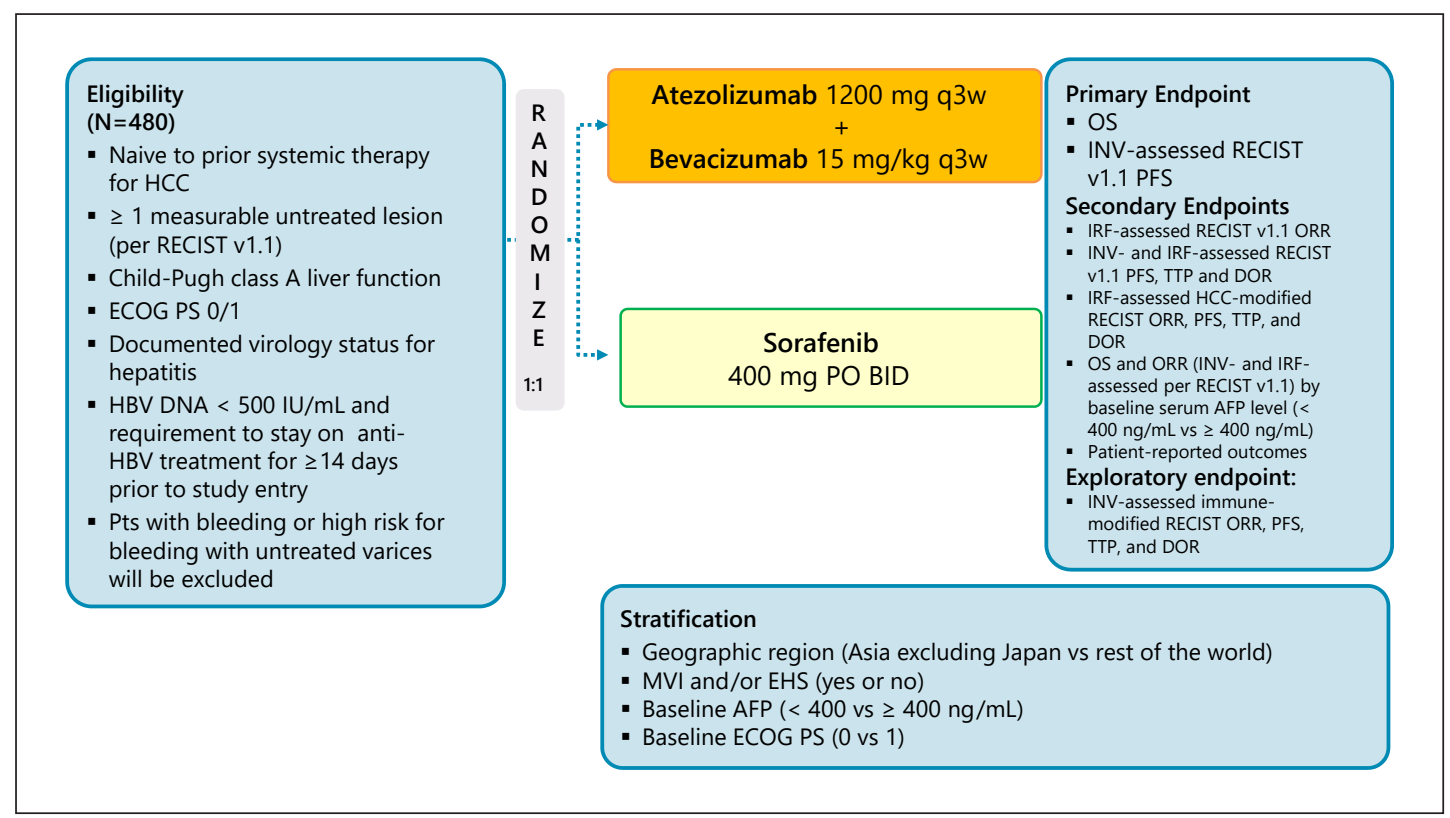

Fig. 11. IMbrave 150: atezolizumab + bevacizumab in first line. Clinical trial information: NCT03434379 [38].

meetings in 2017,2018, and 2019, combination therapy with pembrolizumab and lenvatinib yielded a high response rate of $50-70 \%$ and led to durable responses in patients with solid cancers such as renal cancer, endometrial cancer, and head and neck cancer. For HCC, a phase Ib study of pembrolizumab and lenvatinib is in progress, and a phase III trial (the LEAP002 trial) is also underway (Fig. 10).

The results of five clinical trials of combination immunotherapy with an anti-PD-1/PD-L1 antibody and an anti-CTLA-4 antibody or a molecular targeted agent in HCC were presented at ASCO in 2018, ESMO in 2019, and ASCO in 2019, and at the 2019 annual meeting of the American Association of Cancer Researchers (AACR). The best results were observed in a phase Ib trial of combination therapy with pembrolizumab and lenvatinib. Although only 30 
Kudo: Immuno-Oncology Therapy for Hepatocellular Carcinoma: Current Status and Ongoing Trials

Table 7. Results of immune checkpoint inhibitors and combination cancer immunotherapy in HCC

\begin{tabular}{|c|c|c|c|c|c|c|c|c|}
\hline & \multicolumn{2}{|c|}{ Anti-PD-1 monotherapy } & \multicolumn{4}{|c|}{ Anti-PD-1/PD-L1 plus TKI/anti-VEGF } & \multicolumn{2}{|c|}{ Anti-PD-1/PD-L1 plus anti-CTLA-4 } \\
\hline & $\begin{array}{l}\text { Nivolumab } \\
{[2](n=214)}\end{array}$ & $\begin{array}{l}\text { Pembrolizumab } \\
\text { [7] }(n=278)\end{array}$ & $\begin{array}{l}\text { Pembrolizumab } \\
\text { plus lenvatinib } \\
{[25](n=30)}\end{array}$ & $\begin{array}{l}\text { Atezolizumab } \\
\text { plus bevacizumab } \\
{[27](n=73)}\end{array}$ & $\begin{array}{l}\text { Camrelizumab } \\
\text { plus apatinib } \\
{[28](n=18)}\end{array}$ & $\begin{array}{l}\text { Averumab } \\
\text { plus axitinib } \\
{[29](n=22)}\end{array}$ & $\begin{array}{l}\text { Durvalumab plus } \\
\text { tremelimumab } \\
{[10](n=40)}\end{array}$ & $\begin{array}{l}\text { Nivolumab plus } \\
\text { ipilimumab [13] } \\
(\text { Arm A) }(n=50)\end{array}$ \\
\hline $\begin{array}{l}\text { ORR, } \% \\
(95 \% \mathrm{CI})\end{array}$ & $\begin{array}{l}20 \\
(15-26)\end{array}$ & $\begin{array}{l}18.3 \\
(14.0-23.4)\end{array}$ & $\begin{array}{l}53.3 \\
(34.3-71.7)\end{array}$ & 27 & 38.9 & $\begin{array}{l}13.6 \\
(2.9-34.9)\end{array}$ & 25 & 32 \\
\hline $\begin{array}{l}\text { DCR, \% } \\
(95 \% \mathrm{CI})\end{array}$ & $\begin{array}{l}64 \\
(58-71)\end{array}$ & 62.2 & $\begin{array}{l}90.0 \\
(73.5-97.9)\end{array}$ & 75 & 83.3 & $\begin{array}{l}68.2 \\
(45.1-86.1)\end{array}$ & $\begin{array}{l}57.5 \\
(>16 \text { weeks })\end{array}$ & 54 \\
\hline $\begin{array}{l}\text { PFS, months } \\
(95 \% \mathrm{CI})\end{array}$ & $\begin{array}{l}4.0 \\
(2.9-5.4)\end{array}$ & $\begin{array}{l}3.0 \\
(2.8-4.1)\end{array}$ & $\begin{array}{l}9.7 \\
(7.7-N E)\end{array}$ & $\begin{array}{l}7.5 \\
(0.4-23.9+)\end{array}$ & $\begin{array}{l}7.2 \\
(2.6-\mathrm{NE})\end{array}$ & $\begin{array}{l}5.5 \\
(1.9-7.4)\end{array}$ & NA & NA \\
\hline $\begin{array}{l}\text { OS, months } \\
(95 \% \mathrm{CI})\end{array}$ & $\mathrm{NR}^{1}$ & $\begin{array}{l}13.9 \\
(11.6-16.0)\end{array}$ & $\begin{array}{l}14.6 \\
(9.9-N E)\end{array}$ & NR & NR & $\begin{array}{l}12.7 \\
(8.0-\mathrm{NE})\end{array}$ & NA & $\begin{array}{l}22.8 \\
(9.4-N E)\end{array}$ \\
\hline $\begin{array}{l}\text { DOR, months } \\
\text { (M) }\end{array}$ & $\begin{array}{l}9.9 \\
(8.3-N E)\end{array}$ & $\begin{array}{l}13.8 \\
(1.5-23.6)\end{array}$ & $\begin{array}{l}8.3 \\
(3.8-11.0)\end{array}$ & $\begin{array}{l}\geq 12 \\
(25 \%)\end{array}$ & $\mathrm{NE}$ & $\begin{array}{l}5.5 \\
(3.7-7.3)\end{array}$ & NA & $\begin{array}{l}17.5 \\
(4.6-30.5+)\end{array}$ \\
\hline
\end{tabular}

DCR, disease control rate; DOR, duration of response; NA, not available; NE; not evaluable; NR, not reached; ORR, overall response rate (RECIST 1.1); OS, overall survival; PFS, progression-fee survival. ${ }^{1}$ Nine months: $74 \%$.

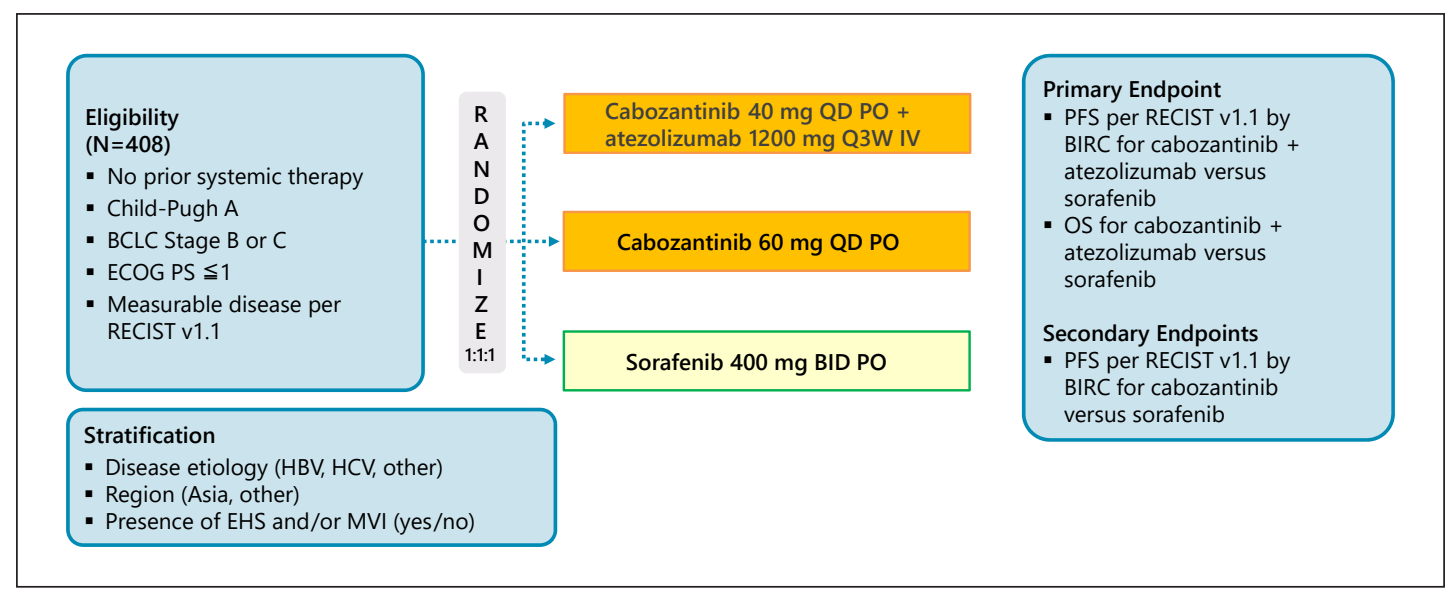

Fig. 12. COSMIC-312: cabozantinib + atezolizumabin in first line (Japan dose not join). Clinical trial information: NCT03755791 [39].

patients were evaluated for safety and efficacy, the ORR per RECIST 1.1 was 53.3\%, the disease control rate was $90.0 \%$, and $6.7 \%$ of patients had PD. PFS was also a favorable 9.7 months. The median duration of response was 8.3 months [25].

The other trial investigated the combination of the PD-L1 antibody atezolizumab with the anti-VEGF antibody bevacizumab. At the time of the 2018 ASCO annual meeting, the response rate among the 23 patients was $65 \%$ using RECIST 1.1 . These high response rates generated considerable excitement in the field [26]. However, the updated data presented at the ESMO congress in 2018 showed that the ORR and disease control rate dropped to 32 and $77 \%$, respectively [27]. Currently, a phase III trial of this combination, IMbravel 150, is ongoing (Fig. 11). The third trial investigated the combination of the anti-PD-1 antibody SHR1210 with apatinib and enrolled 18 patients; the outcomes were promising (response rate, 38.9\%; disease control rate, 83.3\%; PFS, 7.2 months) [28]. At the 2019 ASCO annual meeting, results of a combination trial of avelumab and axitinib were also presented [29] (Table 7).

Another phase III trial, COSMIC-312, is investigating the combination of cabozantinib and atezolizumab (Fig. 12). 


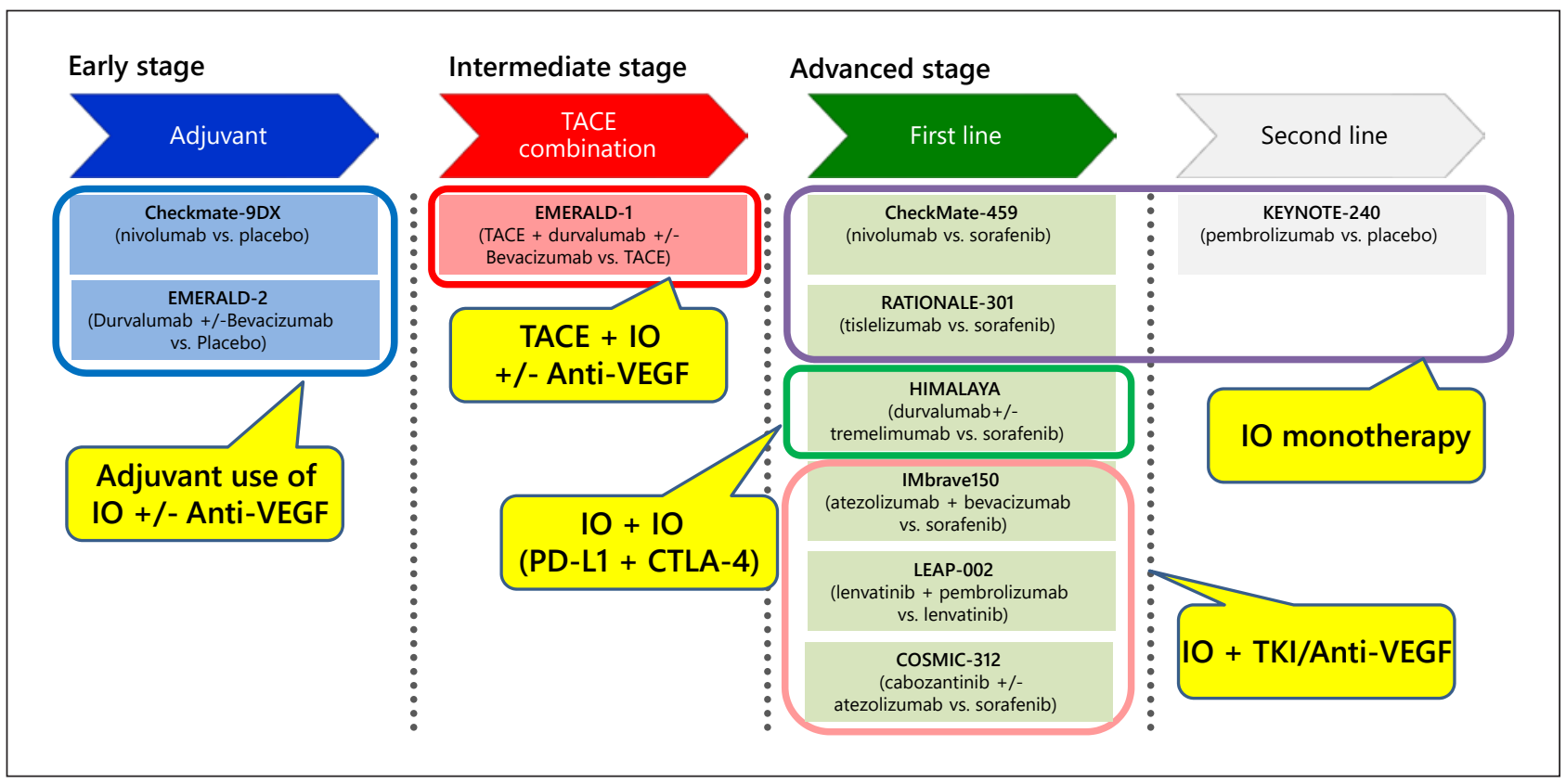

Fig. 13. Immunotherapy in phase III trials.

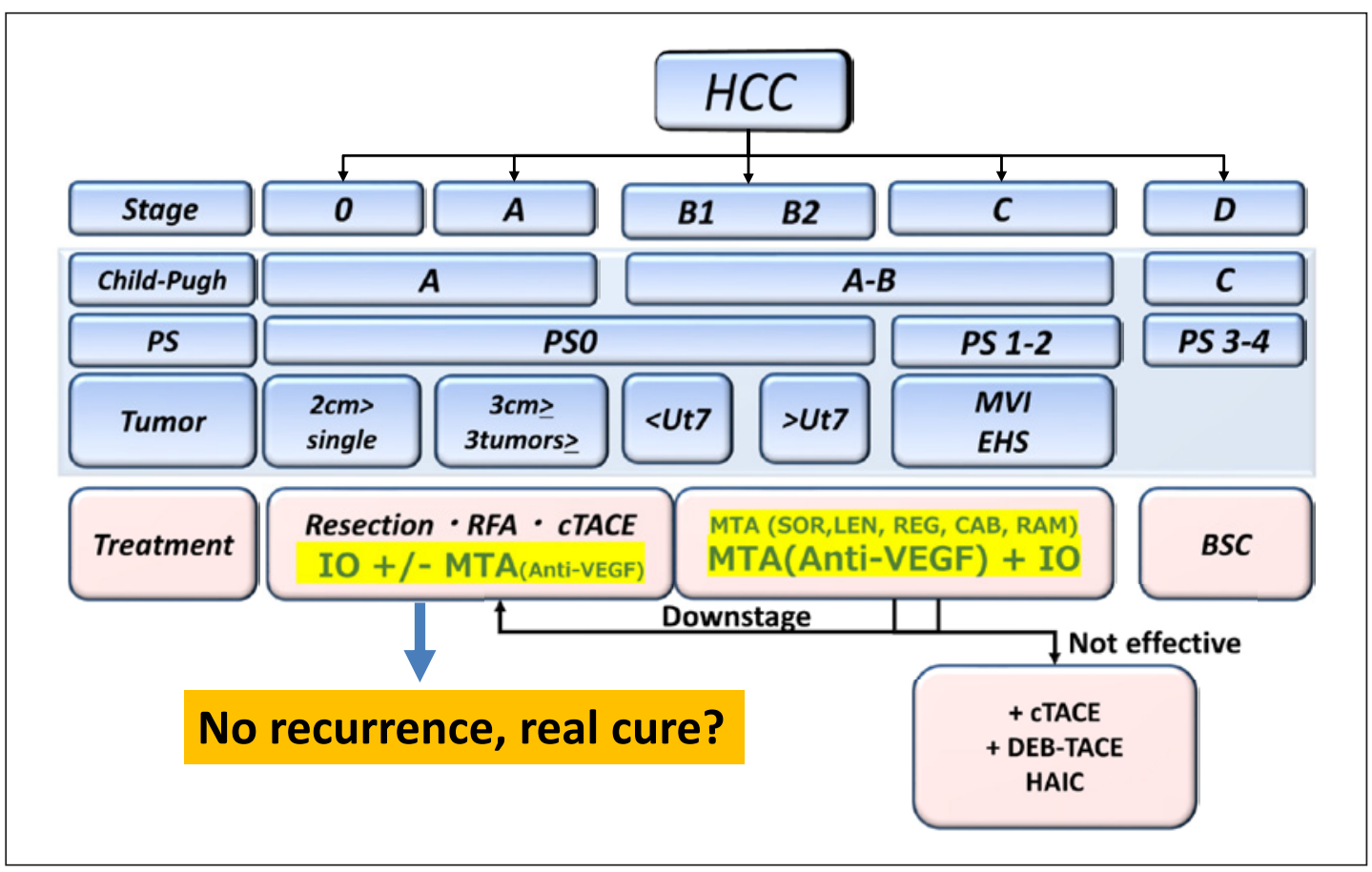

Fig. 14. Future treatment landscape of HCC (within 3-5 years).

Table 7 shows the summary of several combination immunotherapy trials to date. These results could change when these combination treatments are investigated in larger numbers of patients, and results of the phase III IMbrave150 trial of atezolizumab plus bevacizumab and the LEAP-002 trial of lenvatinib plus pembrolizumab are eagerly awaited. Tables 2 and 3 and Figure 13 show ongoing clinical trials of immunotherapies. 


\section{Liver Cancer}

\section{Conclusions}

Sharma and Allison [4, 30] suggest that long-term survival and even cancer cures could be achieved in patients who respond to treatment with immune checkpoint inhibitors. They propose that the high efficacy of combination therapy makes it particularly likely to bring about a "real cure" [4]. It is hoped that checkpoint inhibitors will increase the response to therapy in HCC. In addition, the combination of anti-PD-1/PD-L1 antibodies with molecular targeted agents and other immunotherapies such as anti-CTLA- 4 antibodies may result in a paradigm shift in the treatment of HCC (Fig. 14). The recent emergence of immune checkpoint inhibitors [31] and multiple molecular targeted agents [11], as well as the much more durable responses achieved by combining these drugs [32], may greatly change conventional treatment algorithms for HCC, and further developments in this field are awaited (Fig. 14).

\section{Disclosure Statement}

M. Kudo received lecture fees from Bayer, Eisai, MSD, and Ajinomoto, research grants from Chugai, Otsuka, Takeda, Taiho, Sumitomo Dainippon, Daiichi Sankyo, MSD, Eisai, Bayer, AbbVie, Medico's Hirata, Astellas Pharma, and Bristol-Myers Squibb, and advisory consulting fees from Kowa, MSD, Bristol-Myers Squibb, Bayer, Chugai, Taiho, Eisai, and Ono Pharmaceutical.

\section{References}

1 Chen DS, Mellman I. Oncology meets immunology: the cancer-immunity cycle. Immunity. 2013 Jul;39(1):1-10

2 El-Khoueiry AB, Sangro B, Yau T, Crocenzi TS, Kudo M, Hsu C, et al. Nivolumab in patients with advanced hepatocellular carcinoma (CheckMate 040): an open-label, non-comparative, phase 1/2 dose escalation and expansion trial. Lancet. 2017 Jun;389(10088):2492-502.

3 Merck Sharp \& Dohme. Keytruda (pembrolizumab) prescribing information. March, 2017. https://www. accessdata.fda.gov/drugsatfda_docs/label/2017/125514s015lbl.pdf (accessed May 17, 2018).

4 Sharma P, Allison JP. The future of immune checkpoint therapy. Science. 2015 Apr;348(6230):56-61.

5 Zhu AX, Finn RS, Edeline J, Cattan S, Ogasawara S, Palmer D, et al.; KEYNOTE-224 investigators. Pembrolizumab in patients with advanced hepatocellular carcinoma previously treated with sorafenib (KEYNOTE224): a non-randomised, open-label phase 2 trial. Lancet Oncol. 2018 Jul;19(7):940-52.

6 Carone C, Olivani A, Dalla Valle R, Manuguerra R, Silini EM, Trenti T, et al. Immune Gene Expression Profile in Hepatocellular Carcinoma and Surrounding Tissue Predicts Time to Tumor Recurrence. Liver Cancer. 2018 Sep; 7(3):277-94.

7 Finn RS, Ryoo BY, Merle P, Kudo M, Bouattour M, Lim HY, et al. Results of KEYNOTE-240: phase 3 study of pembrolizumab (Pembro) vs best supportive care (BSC) for second line therapy in advanced hepatocellular carcinoma (HCC). J Clin Oncol. 2019;37(suppl: abstr 4004).

8 Postow MA, Chesney J, Pavlick AC, Robert C, Grossmann K, McDermott D, et al. Nivolumab and ipilimumab versus ipilimumab in untreated melanoma. N Engl J Med. 2015 May;372(21):2006-17.

9 Wolchok JD, Kluger H, Callahan MK, Postow MA, Rizvi NA, Lesokhin AM, et al. Nivolumab plus ipilimumab in advanced melanoma. N Engl J Med. 2013 Jul;369(2):122-33.

10 Kelley RK, Abou-Alfa GK, Bendell JC, Kim TY, Borad MJ, Yong WP, et al. Phase I/II study of durvalumab and tremelimumab in patients with unresectable hepatocellular carcinoma(HCC): Phase I safety and efficacy analyses. J Clin Oncolol. 2017;35(suppl; abstr 4073).

11 Kudo M. Molecular Targeted Agents for Hepatocellular Carcinoma: Current Status and Future Perspectives. Liver Cancer. 2017 Feb;6(2):101-12.

12 Wing K, Onishi Y, Prieto-Martin P, Yamaguchi T, Miyara M, Fehervari Z, et al. CTLA-4 control over Foxp3+ regulatory T cell function. Science. 2008 Oct;322(5899):271-5.

13 Yau T, Kang YK, Kim TY, El-Khoueiry AB, Santoro A, Sangro B, et al. Nivolumab (NIVO) + ipilimumab (IPI) combination therapy in patients (pts) with advanced hepatocellular carcinoma (aHCC): Results from CheckMate 040. J Clin Oncol. 2019;37(suppl: abstr 4012).

14 Duffy AG, Ulahannan SV, Makorova-Rusher O, Rahma O, Wedemeyer H, Pratt D, et al. Tremelimumab in combination with ablation in patients with advanced hepatocellular carcinoma. J Hepatol. 2017 Mar;66(3):545-51.

15 Yoshida H, Shiratori Y, Kudo M, Shiina S, Mizuta T, Kojiro M, et al. Effect of vitamin K2 on the recurrence of hepatocellular carcinoma. Hepatology. 2011 Aug; 54(2):532-40. 
Kudo: Immuno-Oncology Therapy for Hepatocellular Carcinoma: Current Status and Ongoing Trials

16 Bruix J, Takayama T, Mazzaferro V, Chau GY, Yang J, Kudo M, et al.; STORM investigators. Adjuvant sorafenib for hepatocellular carcinoma after resection or ablation (STORM): a phase 3, randomised, double-blind, placebo-controlled trial. Lancet Oncol. 2015 Oct;16(13):1344-54.

17 Mazzaferro V, Romito R, Schiavo M, Mariani L, Camerini T, Bhoori S, et al.; HCC Italian Task Force. Prevention of hepatocellular carcinoma recurrence with alpha-interferon after liver resection in HCV cirrhosis. Hepatology. 2006 Dec;44(6):1543-54.

18 Okita K, Izumi N, Matsui O, Tanaka K, Kaneko S, Moriwaki H, et al.; Peretinoin Study Group. Peretinoin after curative therapy of hepatitis C-related hepatocellular carcinoma: a randomized double-blind placebocontrolled study. J Gastroenterol. 2015 Feb;50(2):191-202.

19 Kudo M. Immune Checkpoint Inhibition in Hepatocellular Carcinoma: Basics and Ongoing Clinical Trials. Oncology. 2017;92 Suppl 1:50-62.

20 Mizukoshi E, Yamashita T, Arai K, Sunagozaka H, Ueda T, Arihara F, et al. Enhancement of tumor-associated antigen-specific T cell responses by radiofrequency ablation of hepatocellular carcinoma. Hepatology. 2013 Apr;57(4):1448-57.

21 Kudo M. Combination Cancer Immunotherapy with Molecular Targeted Agents/Anti-CTLA-4 Antibody for Hepatocellular Carcinoma. Liver Cancer. 2019 Feb;8(1):1-11.

22 Voron T, Marcheteau E, Pernot S, Colussi O, Tartour E, Taieb J, et al. Control of the immune response by proangiogenic factors. Front Oncol. 2014 Apr; 4:70.

23 Tiegs G, Lohse AW. Immune tolerance: what is unique about the liver. J Autoimmun. 2010 Feb;34(1):1-6.

24 Kato T, Bao X, Macgrath S, Tabata K, Hori Y, Tachino S, et al. Lenvatinib mesilate (LEN) enhanced antitumor activity of a PD-1 blockade agent by potentiating Th1 immune response. Ann Oncol. 2016;27(suppl_6):2PD.

25 Ikeda M, Sung MW, Kudo M. Kobayasi, Baron AD, Finn RS, et al. A phase 1b trial of Lenvatinib (LEN) plus pembrolizumab (PEMBRO) in unresectable hepatocellular carcinoma (uHCC): updated results. Presented at the American Association for Cancer Research Annual Meeting on April 3, 2019 in Atlanta, Georgia.

26 Stein S, Pishvaian MJ, Lee MS, et al. Safety and clinical activity of $1 \mathrm{~L}$ atezolizumab + bevacizumab in a phase Ib study in hepatocellular carcinoma (HCC). J Clin Oncol. 2018;36(suppl; abstr 4074).

27 Pishvaian MJ, Lee MS, Ryoo B, Stein S, Lee K, Verret W, et al. Updated safety and clinical activity results from a Phase Ib study of atezolizumab + bevacizumab in hepatocellular carcinoma (HCC). ESMO 2018; October 19-12; Munich, Germany LBA26.

28 Xu JM, Zhang Y, Jia R, Wang Y, Liu R, Zhang G, et al. Anti-programmed death-1 antibody SHR-1210 (S) combined with apatinib (A) for advanced hepatocellular carcinoma (HCC), gastric cancer (GC) or esophagogastric junction (EGJ) cancer refractory to standard therapy: A phase 1 trial. J Clin Oncol. 2018;36(suppl: abstract 4075).

29 Kudo M, Motomura K, Wada Y, Inaba Y, Sakamoto Y, Kurosaki M, et al. First-line avelumab + axitinib in patients with advanced hepatocellular carcinoma: Results from a phase 1b trial (VEGF Liver 100). J Clin Oncol. 2019; 37 (suppl: abstr 4072).

30 Sharma P, Allison JP. Immune checkpoint targeting in cancer therapy: toward combination strategies with curative potential. Cell. 2015 Apr;161(2):205-14.

31 Kudo M. Immune checkpoint blockade in hepatocellular carcinoma: 2017 update. Liver Cancer. 2016 Nov; 6(1):1-12.

32 Kudo M. Combination Cancer Immunotherapy in Hepatocellular Carcinoma. Liver Cancer. 2018 Mar;7(1): 20-7.

33 Sangro B, Park JW, Dela Cruz CM, Anderson J, Lang L, Neely J, Shaw JW, Cheng AL. A randomized, multicenter, phase 3 study of nivolumab vs sorafenib as first-line treatment in patients (pts) with advanced hepatocellular carcinoma (HCC): CheckMate-459. J Clin Oncol. 2016;34(suppl; abstr TPS4147).

34 Finn RS, Chan SL, Zhu AX, Knox JJ, Cheng AL, Siegel AB, Bautista O, Watson P, Kudo M. KEYNOTE-240: Randomized phase III study of pembrolizumab versus best supportive care for second-line advanced hepatocellular carcinoma. J Clin Ocol. 2018;35(suppl; abstr TPS503).

35 Qin S, Finn RS, Kudo M, Meyer T, Vogel A, Ducreux M, Mercade TM, Tomasello G, Boisserie F, Hou J, Li C, Song J, Zhu AX. A phase 3, randomized, open-label, multicenter study to compare the efficacy and safety of tislelizumab, an anti-PD-1 antibody, versus sorafenib as first-line treatment in patients with advanced hepatocellular carcinoma. J Clin Oncol. 2018;36(suppl; abstr TPS3110).

36 Abou-Alfa GK, Chan SL, Furuse J, Galle PR, Kelley RK, Qin S, Armstrong J, Darilay A, Vlahovic G, Negro A, Sangro B. A randomized, multicenter phase 3 study of durvalumab (D) and tremelimumab (T) as first-line treatment in patients with unresectable hepatocellular carcinoma (HCC): HIMALAYA study. J Clin Oncol. 2018;36(suppl; abstr TPS4144).

37 Jimenez Exposito MJ, Akce M, Alvarez JLM, Assenat E, Balart LA, Baron AD, Decaens T, Heurgue-Berlot A, Martin AO, Paik SW, Poulart V, Sehbai AS, Shimada M, Takemura N, Yoon J. CA209-9DX: Phase 3, Randomized, Double-Blind Study of Adjuvant Nivolumab vs Placebo for Patients with Hepatocellular Carcinoma (HCC) at High Risk of Recurrence after Curative Resection or Ablation. 2018. ESMO2018 (abstr TPS4663).

38 Finn RS, Ducreux M, Qin S, Galle PR, Zhu AX, Ikeda M, Kim TY, Xu DZ, Verret W, Liu J, Grossman W, Cheng AL. IMbrave150: A randomized phase III study of $1 \mathrm{~L}$ atezolizumab plus bevacizumab vs sorafenib in locally advanced or metastatic hepatocellular carcinoma. J Clin Oncol. 2018;36(suppl; abstr TPS4141). 
Kudo: Immuno-Oncology Therapy for Hepatocellular Carcinoma: Current Status and Ongoing Trials

39 Kelley RK, Cheng AL, Braiteh FS, Park JW, Benzaghou F, Milwee S, Borgman A, El-Khoueiry AB, Kayali ZK, Zhu AX, Rimassa L. Phase 3 (COSMIC-312) study of cabozantinib (C) in combination with atezolizumab (A) versus sorafenib (S) in patients (pts) with advanced hepatocellular carcinoma (aHCC) who have not received previous systemic anticancer therapy. J Clin Oncol. 2019;37(suppl; abstr TPS4157).

40 Llovet JM, Decaens T, Raoul JL, Boucher E, Kudo M, Chang C, et al. Brivanib in patients with advanced hepatocellular carcinoma who were intolerant to sorafenib or for whom sorafenib failed: results from the randomized phase III BRISK-PS study. J Clin Oncol. 2013 Oct;31(28):3509-16.

41 Zhu AX, Kudo M, Assenat E, Cattan S, Kang YK, Lim HY, et al. Effect of everolimus on survival in advanced hepatocellular carcinoma after failure of sorafenib: the EVOLVE-1 randomized clinical trial. JAMA. 2014 Jul;312(1): 57-67.

42 Kudo M, Moriguchi M, Numata K, Hidaka H, Tanaka H, Ikeda M, et al. S-1 versus placebo in patients with sorafenib-refractory advanced hepatocellular carcinoma (S-CUBE): a randomised, double-blind, multicentre, phase 3 trial. Lancet Gastroenterol Hepatol. 2017 Jun;2(6):407-17.

43 Zhu AX, Park JO, Ryoo BY, Yen CJ, Poon R, Pastorelli D, et al.; REACH Trial Investigators. Ramucirumab versus placebo as second-line treatment in patients with advanced hepatocellular carcinoma following first-line therapy with sorafenib (REACH): a randomised, double-blind, multicentre, phase 3 trial. Lancet Oncol. 2015 Jul;16(7):859-70.

44 Zhu AX, Kang YK, Yen CJ, Finn RS, Galle PR, Llovet JM, et al.; REACH-2 study investigators. Ramucirumab after sorafenib in patients with advanced hepatocellular carcinoma and increased $\alpha$-fetoprotein concentrations (REACH-2): a randomised, double-blind, placebo-controlled, phase 3 trial. Lancet Oncol. 2019 Feb;20(2):28296.

45 Bruix J, Qin S, Merle P, Granito A, Huang YH, Bodoky G, et al.; RESORCE Investigators. Regorafenib for patients with hepatocellular carcinoma who progressed on sorafenib treatment (RESORCE): a randomised, doubleblind, placebo-controlled, phase 3 trial. Lancet. 2017 Jan;389(10064):56-66.

46 Abou-Alfa GK, Meyer T, Cheng AL, El-Khoueiry AB, Rimassa L, Ryoo BY, et al. Cabozantinib in Patients with Advanced and Progressing Hepatocellular Carcinoma. N Engl J Med. 2018 Jul;379(1):54-63. 